\title{
ON THE NUMBER OF EXCEPTIONAL POINTS OF HOLOMORPHIC CURVES AND THE DEFECT RELATION FOR HOLOMORPHIC CURVES
}

\author{
Nobushige TodA
}

\begin{abstract}
Let $X^{n}(2)$ be a subset of $\boldsymbol{C}^{n+1} \backslash\{\boldsymbol{0}\}$ any two elements of which are not propotional. We estimate the number of exceptional points in $X^{n}(2)$ for several holomorphic curves and we consider the defect relation for holomorphic curves. We shall give an example for which the defect relation is extremal and then give some holomorphic curves for which the defect relation is not extremal over $X^{n}(2)$. Another defect relation is also considered.
\end{abstract}

\section{Introduction}

Let $f=\left[f_{1}, \ldots, f_{n+1}\right]$ be a holomorphic curve from $\boldsymbol{C}$ into the $n$-dimensional complex projective space $P^{n}(\boldsymbol{C})$ with a reduced representation

$$
\left(f_{1}, \ldots, f_{n+1}\right): \boldsymbol{C} \rightarrow \boldsymbol{C}^{n+1} \backslash\{\boldsymbol{0}\},
$$

where $n$ is a positive integer. We put

$$
\|f(z)\|=\left(\left|f_{1}(z)\right|^{2}+\cdots+\left|f_{n+1}(z)\right|^{2}\right)^{1 / 2}
$$

and the characteristic function of $f$ is given as follows (see [14]):

$$
T(r, f)=\frac{1}{2 \pi} \int_{0}^{2 \pi} \log \left\|f\left(r e^{i \theta}\right)\right\| d \theta-\log \|f(0)\| .
$$

It is known ([1]) that for $U(z)=\max _{1 \leq j \leq n+1}\left|f_{j}(z)\right|$

$$
T(r, f)=\frac{1}{2 \pi} \int_{0}^{2 \pi} \log U\left(r e^{i \theta}\right) d \theta+O(1) .
$$

We suppose throughout the paper that $f$ is transcendental; that is to say,

$$
\lim _{r \rightarrow \infty} T(r, f) / \log r=\infty \text {. }
$$

2010 Mathematics Subject Classification. 30D35.

Key words and phrases. Holomorphic curves, Number of exceptional points, Defect relation. Received February 26, 2013. 
The order $\rho_{f}$ of $f$ is given as follows:

$$
\rho_{f}=\limsup _{r \rightarrow \infty} \log T(r, f) / \log r .
$$

For $\boldsymbol{a}=\left(a_{1}, \ldots, a_{n+1}\right) \in \boldsymbol{C}^{n+1} \backslash\{\boldsymbol{0}\}$, we put

$$
(\boldsymbol{a}, f)=a_{1} f_{1}+\cdots+a_{n+1} f_{n+1}
$$

and we use the standard notaitions $N(r, \boldsymbol{a}, f), N_{n}(r, \boldsymbol{a}, f), \delta(\boldsymbol{a}, f), \delta_{n}(\boldsymbol{a}, f)$ as usual (see e.g. [12, Introduction]).

Let $S(r, f)$ be any quantity satisfying

$$
S(r, f)=o(T(r, f)) \quad(r \rightarrow \infty ; r \notin E),
$$

where $E$ is a subset of $(0, \infty)$ of finite linear measure.

We suppose throughout the paper that $f$ is linearly non-degenerate over $\boldsymbol{C}$. Namely, the Wronskian of the functions $f_{1}, \ldots, f_{n+1}$ is not identically equal to zero. Let $X$ be a subset of $\boldsymbol{C}^{n+1} \backslash\{\boldsymbol{0}\}$ in $N$-subgeneral position and we put

(a) $D^{1}=\left\{\boldsymbol{a} \in X \mid \delta_{n}(\boldsymbol{a}, f)=1\right\}$;

(b) $P=\{\boldsymbol{a} \in X \mid(\boldsymbol{a}, f)$ has at most a finite number of zeros in $|z|<\infty\}$;

(c) $M^{1}=\{\boldsymbol{a} \in X \mid(\boldsymbol{a}, f)$ has no zeros in $|z|<\infty\}$,

(d) $B=\left\{\boldsymbol{a} \in X \mid \lim \sup _{r \rightarrow \infty} \log N_{n}(r, \boldsymbol{a}, f) / \log r<\rho_{f}\right\}$,

where $N$ is an integer such that $N \geq n$. We say that $a \in B$ is Borel exceptional for $f$. Then, $M^{1} \subset P \subset D^{1}$ and it is known that

$$
\# P \leq \# D^{1} \leq 2 N-n+1, \quad B \nsubseteq D^{1}, \quad D^{1} \nsubseteq B \quad \text { and } \quad \# M^{1} \leq N+N / n
$$

(see e.g. [3], [9], [13]).

In this paper, with the set $X$ in $N$-subgeneral position, we consider the following subsets of $\boldsymbol{C}^{n+1} \backslash\{\boldsymbol{0}\}$, which are not always in subgeneral position. For $k=1, \ldots, n+1$, let $X^{n}(k)$ be a subset of $\boldsymbol{C}^{n+1} \backslash\{\mathbf{0}\}$, satisfying that any $k$ elements of which are linearly independent. In particular, our main one is $X^{n}(2)$ in this paper. From the definition it satisfies

(*) For any $\boldsymbol{a}, \boldsymbol{b} \in X^{n}(2), \boldsymbol{a} \neq \alpha \boldsymbol{b}(\alpha \in \boldsymbol{C})$.

The set $X^{n}(n+1)$ is in general position.

The first purpose of this paper is to estimate the number of elements of the following subsets of $X^{n}(2)$.

Definition 1.A. (1) $\mathscr{D}^{1}=\left\{\boldsymbol{a} \in X^{n}(2) \mid \delta_{n}(\boldsymbol{a}, f)=1\right\}$

(2) $\mathscr{P}=\left\{\boldsymbol{a} \in X^{n}(2) \mid(\boldsymbol{a}, f)\right.$ has at most a finite number of zeros in $\left.|z|<\infty\right\}$;

(3) $\mathscr{M}^{1}=\left\{\boldsymbol{a} \in X^{n}(2) \mid(\boldsymbol{a}, f)\right.$ has no zeros in $\left.|z|<\infty\right\}$;

(4) $\mathscr{B}=\left\{\boldsymbol{a} \in X^{n}(2) \mid \lim \sup _{r \rightarrow \infty} \log N_{n}(r, \boldsymbol{a}, f) / \log r<\rho_{f}\right\}$.

The main tool is the fundamental inequality of $\mathrm{H}$. Cartan ([1]). Some applications of our results are given. We estimate them when $f$ is linearly nondegenerate over a function field

Note 1.1. An interesting result is given in a more delicate situation ([2, Theorem 5.2]). 
The second purpose is to estimate $\#\left\{D^{1} \cup B\right\}$ in $X$ and to estimate $\#\left\{\mathscr{D}^{1} \cup \mathscr{B}\right\}$ in $X^{n}(2)$ under a condition.

The last purpose is to give the defect relation of holomorphic curves, one of which is extremal and others are not extremal.

As is cited above the main tool in this paper is the fundamental inequality of H. Cartan ([1]) for holomorphic curves. We also use its generalization by Nochka ([7]).

\section{Number of exceptional points of holomorphic curves}

Let $f, X, X^{n}(2), N, n$ etc. be as in Section 1. First of all we give the main tool of this paper, the fundamental inequality by H. Cartan ([1]).

2-1. Lemma. We shall give some lemmas for later use in this section.

Lemma 2.1 ([1]). For any q elements $\boldsymbol{a}_{j}(j=1, \ldots, q)$ of $\boldsymbol{C}^{n+1} \backslash\{\boldsymbol{0}\}$ in general position $(n+2 \leq q<\infty)$, we have the following inequalities:

(I) $(q-n-1) T(r, f) \leq \sum_{j=1}^{q} N_{n}\left(r, \boldsymbol{a}_{j}, f\right)+S(r, f)$.

(II) $\sum_{j=1}^{q} \delta_{n}\left(\boldsymbol{a}_{j}, f\right) \leq n+1$.

Note 2.1. When $\rho_{f}$ is finite, the error term $S(r, f)$ can be replaced by $O(\log r)$ without exceptional intervals (see the proof of (I) and [4, Theorem 2.2(i)]).

We put

$$
\mathscr{M}_{f}=\{\varphi \mid \text { meromorphic in }|z|<\infty ; T(r, \varphi)=S(r, f)\}
$$

and when $0<\rho_{f}<\infty$

$$
\mathscr{M}_{o}=\left\{\varphi \mid \text { meromorphic in }|z|<\infty ; \rho_{\varphi}<\rho_{f}\right\},
$$

where $\rho_{\varphi}$ is the order of $\varphi$.

We know that the sets $\mathscr{M}_{f}$ and $\mathscr{M}_{o}$ are fields. We denote by $\mathscr{M}$ the field $\mathscr{M}_{f}$, any subfield of $\mathscr{M}_{f}$ or $\mathscr{M}_{o}$ and from now on we suppose that the curve $f=\left[f_{1}, \ldots, f_{n+1}\right]$ is linearly non-degenerate over $\mathscr{M}$.

We note that $\varphi^{\prime} \in \mathscr{M}_{f}$ for $\varphi \in \mathscr{M}_{f}$ because $T\left(r, \varphi^{\prime}\right) \leq 2 T(r, \varphi)+S(r, \varphi)$.

Lemma 2.2. Let $\boldsymbol{a}_{j}=\left(a_{j 1}, \ldots, a_{j n+1}\right)(j=1, \ldots, v ; 2 \leq v \leq n+1)$ be $v$ linearly independent vectors in $\boldsymbol{C}^{n+1}$, let $\boldsymbol{a}$ be a vector in $\boldsymbol{C}^{n+1}$ such that

$$
\boldsymbol{a}=\sum_{j=1}^{v} c_{j} \boldsymbol{a}_{j} \quad\left(c_{j} \neq 0 \in \boldsymbol{C}\right)
$$

and we put

$$
F_{j}=\left(\boldsymbol{a}_{j}, f\right) \quad(j=1, \ldots, v), \quad F_{o}=(\boldsymbol{a}, f) .
$$


Let $g$ be an entire function such that the functions $F_{1} / g, \ldots, F_{v} / g$ are entire functions without common zeros and put $F=\left[F_{1} / g, \ldots, F_{v} / g\right]$.

Then, we have the followings:

(I) The functions $F_{1}, \ldots, F_{v}$ are linearly independent over $\mathscr{M}$.

In particular,

$$
F_{j_{1}} / F_{j_{2}} \notin \mathscr{M} \quad\left(1 \leq j_{1} \neq j_{2} \leq v\right) .
$$

(II) (i) $T(r, F) \leq T(r, f)+O(1)(r \geq 1)$.

(ii) $T\left(r, F_{j_{1}} / F_{j_{2}}\right) \leq T(r, F)+O(1)\left(1 \leq j_{1} \neq j_{2} \leq v\right)$.

(III) If $F$ is transcendental,

$$
T(r, F) \leq \sum_{j=1}^{v} N_{n}\left(r, \boldsymbol{a}_{j}, f\right)+N_{n}(r, \boldsymbol{a}, f)+S(r, F) .
$$

Proof. (I) Suppose that $\sum_{j=1}^{v} g_{j} F_{j}=0\left(g_{j} \in \mathscr{M}\right)$. Then, as $f_{1}, \ldots, f_{n+1}$ are linearly independent over $\mathscr{M}$, we obtain the equation

$$
\sum_{j=1}^{v} a_{j k} g_{j}=0 \quad(k=1, \ldots, n+1) .
$$

We put $\boldsymbol{b}_{k}=\left(a_{1 k}, a_{2 k}, \ldots, a_{v k}\right)(1 \leq k \leq n+1)$. Then,

$$
\left({ }^{t} \boldsymbol{a}_{1}, \ldots,{ }^{t} \boldsymbol{a}_{v}\right)=\left(\begin{array}{c}
\boldsymbol{b}_{1} \\
\vdots \\
\boldsymbol{b}_{n+1}
\end{array}\right) \text { and } \operatorname{rank}=\left(\begin{array}{c}
\boldsymbol{b}_{1} \\
\vdots \\
\boldsymbol{b}_{n+1}
\end{array}\right)=v
$$

since $\boldsymbol{a}_{1}, \ldots, \boldsymbol{a}_{v}$ are linearly independent, so that there are $v$ linearly independent vectors $\boldsymbol{b}_{k_{1}}, \ldots, \boldsymbol{b}_{k_{v}}$ in $\left\{\boldsymbol{b}_{1}, \ldots, \boldsymbol{b}_{n+1}\right\}$.

From the equation

$$
\sum_{j=1}^{v} a_{j k_{i}} g_{j}=0 \quad(i=1, \ldots, v),
$$

we obtain that $g_{1}=0, \ldots, g_{v}=0$, so that $F_{1}, \ldots, F_{v}$ are linearly independent over M.

(II) (i) From the definition we have the relation

$$
\begin{aligned}
\log \|F\| & =\log \left(\sum_{j=1}^{v}\left|F_{j} / g\right|^{2}\right)^{1 / 2}=\log \left(\sum_{j=1}^{v}\left|F_{j}\right|^{2}\right)^{1 / 2}-\log |g| \\
& \leq \log \|f\|-\log |g|+O(1),
\end{aligned}
$$

so that

$$
T(r, F) \leq T(r, f)-N(r, 1 / g)+O(1) \leq T(r, f)+(1) \quad(r \geq 1) .
$$


(ii) From [1, p. 10], we have the inequality

$$
T(r, F)+O(1) \geq T\left(r, \frac{F_{j_{1}} / g}{F_{j_{2}} / g}\right)=T\left(r, F_{j_{1}} / F_{j_{2}}\right)
$$

(III) We put $\boldsymbol{c}=\left(c_{1}, \ldots, c_{v}\right)$. Then, we have that $\boldsymbol{e}_{1}, \ldots, \boldsymbol{e}_{v}, \boldsymbol{c} \in \boldsymbol{C}^{v}$ are in general position since $c_{j} \neq 0(j=1, \ldots, v)$ and that for $F_{o}=(\boldsymbol{a}, f)$

$$
F_{o}=\sum_{j=1}^{v} c_{j} F_{j}
$$

We apply Lemma 2.1(I) to (2.1) for $n=v-1, F$ and $\left\{\boldsymbol{e}_{1}, \ldots, \boldsymbol{e}_{v}, \boldsymbol{c}\right\}$. Then, we obtain the inequality

$$
T(r, F) \leq \sum_{j=1}^{v} N_{v-1}\left(r, \boldsymbol{e}_{j}, F\right)+N_{v-1}(r, \boldsymbol{c}, F)+S(r, F) .
$$

Here, from the definition of counting function we have the relations for $r \geq 1$

$$
N_{v-1}\left(r, \boldsymbol{e}_{j}, F\right) \leq N_{n}\left(r, \boldsymbol{a}_{j}, f\right) \quad(j=1, \ldots, v) ; \quad N_{v-1}(r, \boldsymbol{c}, F) \leq N_{n}(r, \boldsymbol{a}, f)
$$

since $v-1 \leq n$, so that we obtain (III).

We denote by $\mathscr{K}$ any one of $\mathscr{D}^{1}, \mathscr{P}, \mathscr{M}^{1}$ and $\mathscr{B}$. We suppose that $f$ is linearly non-degenerate over $\mathscr{M}$, where $\mathscr{M}=\mathscr{M}_{f}$ when $\mathscr{K}=\mathscr{D}^{1}, \mathscr{M}=\boldsymbol{C}(z)$ when $\mathscr{K}=\mathscr{P}, \mathscr{M}=\boldsymbol{C}$ when $\mathscr{K}=\mathscr{M}^{1}$ and $\mathscr{M}=\mathscr{M}_{o}$ when $\mathscr{K}=\mathscr{B}$. Then, we obtain the following.

Lemma 2.3. Suppose that $\mathscr{K} \neq \phi$. Then, for $1 \leq k \leq \min \{\# \mathscr{K}, n+1\}$, any $k$ vectors in $\mathscr{K}$ are linearly independent over $\boldsymbol{C}$.

Proof. (i) When $k=1$. This is trivial since $\mathscr{K} \subset X^{n}(2) \subset \boldsymbol{C}^{n+1} \backslash\{\boldsymbol{0}\}$.

(ii) When $k=2$. This is also trivial since $\mathscr{K}$ satisfies $(*)$ in Introduction.

(iii) For $2 \leq k \leq \min \{\# \mathscr{K}, n+1\}-1$, suppose that any $k$ vectors in $\mathscr{K}$ are linearly independent over $\boldsymbol{C}$. We prove that any $k+1$ vectors in $\mathscr{K}$ are linearly independent over $\boldsymbol{C}$.

Suppose to the contrary that there are $k+1$ vectors in $\mathscr{K}: \boldsymbol{a}_{1}, \ldots, \boldsymbol{a}_{k}, \boldsymbol{a}$ which are linearly dependent over $\boldsymbol{C}$. Then, from the hypothesis of the induction we can write

$$
\boldsymbol{a}=\sum_{j=1}^{k} c_{j} \boldsymbol{a}_{j} \quad\left(c_{j} \neq 0,1 \leq j \leq k\right),
$$

from which we obtain the relation

$$
(\boldsymbol{a}, f)=\sum_{j=1}^{k} c_{j}\left(\boldsymbol{a}_{j}, f\right)
$$


We put

$$
F_{j}=\left(\boldsymbol{a}_{j}, f\right) \quad(j=1, \ldots, k) \quad \text { and } \quad F_{o}=(\boldsymbol{a}, f) .
$$

Then, we obtain that from Lemma $2.2(\mathrm{I}) F_{1}, \ldots, F_{k}$ are linearly independent over $\mathscr{M}$ and that from (2.2)

$$
F_{o}=\sum_{j=1}^{k} c_{j} F_{j} \quad\left(c_{j} \neq 0\right) .
$$

Let $g$ be an entire function such that the functions $F_{1} / g, \ldots, F_{k} / g$ are entire functions without common zeros, where $g$ is polynomial when $\mathscr{K}=\mathscr{P}$ and $g=1$ when $\mathscr{K}=\mathscr{M}^{1}$. We put

$$
F=\left[F_{1} / g, \ldots, F_{k} / g\right] .
$$

Then, $F$ is transcendental from Lemma $2.2(\mathrm{I})$ and (II)(ii) when $\mathscr{K}=\mathscr{D}^{1}, \mathscr{P}$ or $\mathscr{B}$. When $\mathscr{K}=\mathscr{M}^{1}$, from Lemma $2.2(\mathrm{I}) F_{j_{1}} / F_{j_{2}}$ is not constant $\left(1 \leq j_{1} \neq j_{2} \leq k\right)$. As $F_{j_{1}}, F_{j_{2}}$ have no zero, $F_{j_{1}} / F_{j_{2}}$ has neither zero nor pole. This implies that $F_{j_{1}} / F_{j_{2}}$ is transcendental, so that $F$ is transcendental from Lemma 2.2(II)(ii). Then, from (2.3) and Lemma 2.2(III) we obtain the inequality

$$
T(r, F) \leq \sum_{j=1}^{k} N_{n}\left(r, \boldsymbol{a}_{j}, f\right)+N_{n}(r, \boldsymbol{a}, f)+S(r, F) .
$$

(a) When $\mathscr{K}=\mathscr{D}^{1}$, from Lemma 2.2(I), (II)(i),(ii) and (2.4) we obtain the inequality

$$
\begin{aligned}
0< & \limsup _{r \rightarrow \infty, r \notin E} \frac{T\left(r, F_{2} / F_{1}\right)}{T(r, f)} \leq \sum_{j=1}^{k} \limsup _{r \rightarrow \infty} \frac{N_{n}\left(r, \boldsymbol{a}_{j}, f\right)}{T(r, f)} \\
& +\limsup _{r \rightarrow \infty} \frac{N_{n}(r, \boldsymbol{a}, f)}{T(r, f)}+\limsup _{r \rightarrow \infty, r \notin E} \frac{S(r, F)}{T(r, f)}=0
\end{aligned}
$$

since $\boldsymbol{a}_{j}, \boldsymbol{a} \in \mathscr{D}^{1}$. This is a contradiction. This means that any $k+1$ vectors in $\mathscr{D}^{1}$ must be linearly independent over $\boldsymbol{C}$.

(b) When $\mathscr{K}=\mathscr{P}$, from (2.4) we obtain the inequality

$$
T(r, F) \leq O(\log r)+S(r, F)=S(r, F)
$$

since $\boldsymbol{a}_{j}, \boldsymbol{a} \in \mathscr{P}$ and $F$ is transcendental. This is a contradiction. This means that any $k+1$ vectors in $\mathscr{P}$ must be linearly independent over $\boldsymbol{C}$.

(c) When $\mathscr{K}=\mathscr{M}^{1}$, from (2.4) we obtain the inequality $T(r, F) \leq S(r, F)$ since $\boldsymbol{a}_{j}, \boldsymbol{a} \in \mathscr{M}^{1}$. This is a contradiction since $F$ is transcendental. This means that any $k+1$ vectors in $\mathscr{M}^{1}$ must be linearly independent over $\boldsymbol{C}$. 
(d) When $\mathscr{K}=\mathscr{B}$, from (2.4) and Note 2.1 we obtain the inequality

$$
T(r, F) \leq \sum_{j=1}^{k} N_{n}\left(r, \boldsymbol{a}_{j}, f\right)+N_{n}(r, \boldsymbol{a}, f)+O(\log r),
$$

since $\rho_{F} \leq \rho_{f}<\infty$. As $\rho_{F}=\rho_{f}$ from Lemma 2.2(I) and (II)(i),(ii), we have the inequality

$$
\begin{aligned}
\rho_{f} & =\limsup _{r \rightarrow \infty} \frac{\log T(r, F)}{\log r} \\
& \leq \limsup _{r \rightarrow \infty} \frac{\log \left(\sum_{j=1}^{k} N_{n}\left(r, \boldsymbol{a}_{j}, f\right)+N_{n}(r, \boldsymbol{a}, f)+O(\log r)\right)}{\log r}<\rho_{f}
\end{aligned}
$$

since $\boldsymbol{a}_{j}, \boldsymbol{a} \in \mathscr{B}$. This is a contradiction. This means that any $k+1$ vectors in $\mathscr{B}$ must be linearly independent over $\boldsymbol{C}$.

From (i), (ii) and (iii) we obtain our lemma.

Lemma 2.4 (see [4, p. 24]). Let $\varphi(z)$ be transcendental meromorphic with at most a finite number of zeros and poles in $|z|<\infty$. Then,

$$
\liminf _{r \rightarrow \infty} T(r, \varphi) / r>0 \text {. }
$$

Last of all in this section we shall give the following curve for later use.

Lemma 2.5. Let $f_{o}=\left[1, e^{z}, \ldots, e^{n z}\right]$. Then, $f_{o}$ is linearly independent over $\mathscr{M}_{f_{o}}$.

Proof. We first note that $f_{o}$ is transcendental since $T\left(r, f_{o}\right) \sim(n / \pi) r$ and that

$$
T\left(r, e^{(i-j) z}\right) \sim \frac{|i-j|}{\pi} r \quad(i \neq j) .
$$

(i) When $n=1$. We put

$$
g_{1}+g_{2} e^{z}=0 \quad\left(g_{1}, g_{2} \in \mathscr{M}_{f_{o}}\right) .
$$

Suppose that $g_{2} \neq 0$. Then, $e^{z}=-g_{1} / g_{2}$, and we have $T\left(r, e^{z}\right)=$ $T\left(r, g_{1} / g_{2}\right)$, so that we have the relation

$$
0<\limsup _{r \rightarrow \infty, r \notin E} \frac{T\left(r, e^{z}\right)}{T\left(r, f_{o}\right)}=\limsup _{r \rightarrow \infty, r \notin E} \frac{T\left(r, g_{1} / g_{2}\right)}{T\left(r, f_{o}\right)}=0,
$$

which is a contradiction. This means that $g_{2}$ must be equal to zero and we have that $g_{1}=0$.

(ii) We suppose that this lemma holds for an integer $k \geq 2$ :

$$
\sum_{j=1}^{k} g_{j} e^{(j-1) z}=0 \quad\left(g_{j} \in \mathscr{M}_{f_{o}}\right) \Rightarrow g_{j}=0 \quad(j=1, \ldots, k)
$$


and we prove that if

$$
\sum_{j=1}^{k+1} g_{j} e^{(j-1) z}=0 \quad\left(g_{j} \in \mathscr{M}_{f_{o}}, j=1, \ldots, k+1\right),
$$

then $g_{j}=0(j=1, \ldots, k+1)$.

Proof. (a) When $g_{1}=0$. In this case, from (2.5) we have the relation

$$
\sum_{j=2}^{k+1} g_{j} e^{(j-2) z}=0
$$

so that from the hypothesis of the induction $g_{2}=0, \ldots, g_{k+1}=0$.

(b) Suppose that $g_{1} \neq 0$. Then, there exists at least one $j(2 \leq j \leq k+1)$ such thst $g_{j} \neq 0$. If $g_{j}=0$ for all $j(2 \leq j \leq k+1), g_{1}=0$ from (2.5).

From (2.5), we obtain

$$
1+\sum_{j=2}^{k+1} \frac{g_{j}}{g_{1}} e^{(j-1) z}=0 .
$$

Here, $g_{j} / g_{1} \in \mathscr{M}_{f_{o}}(j=2, \ldots, k+1)$. Differentiating (2.6), we obtain

$$
\sum_{j=2}^{k+1}\left\{\left(\frac{g_{j}}{g_{1}}\right)^{\prime}+(j-1) \frac{g_{j}}{g_{1}}\right\} e^{(j-1) z}=0 .
$$

Now, $g_{j} / g_{1} \in \mathscr{M}_{f_{o}},\left(g_{j} / g_{1}\right)^{\prime} \in \mathscr{M}_{f_{o}}(j=2, \ldots, k+1)$ so that from the hypothesis of the induction we have the relations

$$
\left(\frac{g_{j}}{g_{1}}\right)^{\prime}+(j-1) \frac{g_{j}}{g_{1}}=0 \quad(j=2, \ldots, k+1) .
$$

For $j(2 \leq j \leq k+1)$ such that $g_{j} \neq 0$, the function $g_{j} / g_{1}$ satisfies the differential equation

$$
\left(g_{j} / g_{1}\right)^{\prime} /\left(g_{j} / g_{1}\right)=-(j-1)
$$

from which we obtain

$$
g_{j} / g_{1}=c e^{-(j-1) z} \quad(c \neq 0) \quad \text { and } \quad T\left(r, \frac{g_{j}}{g_{1}}\right)=T\left(r, c e^{-(j-1) z}\right) .
$$

From this relation we have that

$$
0=\limsup _{r \rightarrow \infty, r \notin E} \frac{T\left(r, g_{j} / g_{1}\right)}{T\left(r, f_{o}\right)}=\limsup _{r \rightarrow \infty, r \notin E} \frac{T\left(r, c e^{-(j-1) z}\right)}{T\left(r, f_{o}\right)}>0,
$$

which is a contradiction. This implies that $g_{1}$ must be equal to 0 . Then, we obtain that $g_{2}=0, \ldots, g_{k+1}=0$ as in (a).

This means that $f_{o}$ is linearly non-degenerate over $\mathscr{M}_{f_{o}}$. 
2-2. Theorem. Let $f, n, X, X^{n}(2), \mathscr{D}^{1}$ etc. be as in Section 1.

THEOREM 2.1.

(I) Suppose that $f$ is linearly non-degenerate over $\mathscr{M}_{f}$. Then, $\# \mathscr{D}^{1} \leq n+1$.

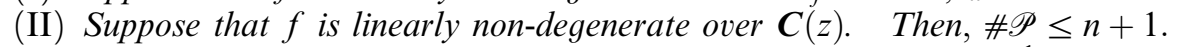

(III) Suppose that $f$ is linearly non-degenerate over $C$. Then, $\# \mathscr{M}^{1} \leq n+1$.

(IV) Suppose that $f$ is linearly non-degenerate over $\mathscr{M}_{o}$ and $0<\rho_{f}<\infty$.

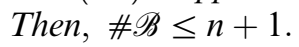

Proof. We denote by $\mathscr{K}$ any one of $\mathscr{D}^{1}, \mathscr{P}, \mathscr{M}^{1}$ and $\mathscr{B}$. We suppose that $f$ is linearly non-degenerate over $\mathscr{M}$, where $\mathscr{M}=\mathscr{M}_{f}$ when $\mathscr{K}=\mathscr{D}^{1}, \mathscr{M}=\boldsymbol{C}(z)$ when $\mathscr{K}=\mathscr{P}, \mathscr{M}=C$ when $\mathscr{K}=\mathscr{M}^{1}$ and $\mathscr{M}=\mathscr{M}_{o}$ when $\mathscr{K}=\mathscr{B}$.

Suppose that $\# \mathscr{K}>n+1$. Then, $\mathscr{K}$ contains at least $n+2$ elements. Let $\boldsymbol{a}_{1}, \ldots, \boldsymbol{a}_{n+2}$ be in $\mathscr{K}$. Then, from Lemma 2.3 , any $n+1$ vectors of $\boldsymbol{a}_{1}, \ldots, \boldsymbol{a}_{n+2}$ are linearly independent over $\boldsymbol{C}$. This means that $\boldsymbol{a}_{1}, \ldots, \boldsymbol{a}_{n+2}$ are in general position in $\boldsymbol{C}^{n+1}$.

(i) When $\mathscr{K}=\mathscr{D}^{1}, \mathscr{P}$ or $\mathscr{M}^{1}$. As $f$ is transcendental in this paper, from lemma $2.1($ II) we have the inequality

$$
\sum_{j=1}^{n+2} \delta_{n}\left(\boldsymbol{a}_{j}, f\right) \leq n+1 .
$$

On the other hand as $\delta_{n}\left(\boldsymbol{a}_{j}, f\right)=1(j=1, \ldots, n+2)$ in any case, we have the equality

$$
\sum_{j=1}^{n+2} \delta_{n}\left(\boldsymbol{a}_{j}, f\right)=n+2,
$$

which is a contradiction. This means that $\# \mathscr{K} \leq n+1$.

(ii) When $\mathscr{K}=\mathscr{B}$. From Lemma 2.1 and Note 2.1, we have the inequality

$$
T(r, f) \leq \sum_{j=1}^{n+2} N_{n}\left(r, \boldsymbol{a}_{j}, f\right)+O(\log r),
$$

from which we obtain the inequality

$$
\rho_{f} \leq \limsup _{r \rightarrow \infty} \frac{\log \left(\sum_{j=1}^{n+2} N_{n}\left(r, \boldsymbol{a}_{j}, f\right)+O(\log r)\right)}{\log r}<\rho_{f}
$$

since $\boldsymbol{a}_{j} \in \mathscr{B}(j=1, \ldots, n+2)$, which is a contradiction.

From (i) and (ii) we have our theorem.

Corollary 2.1. Let $\boldsymbol{a}_{1}, \ldots, \boldsymbol{a}_{n+2}$ be any $n+2$ elements in $X^{n}(2)$.

(I) If $f$ is linearly non-degenerate over $\mathscr{M}_{f}$, then

$$
\limsup _{r \rightarrow \infty} \frac{\sum_{j=1}^{n+2} N_{n}\left(r, \boldsymbol{a}_{j}, f\right)}{T(r, f)}>0 .
$$


(II) If $f$ is linearly non-degenerate over $\boldsymbol{C}(z)$, then

$$
\lim _{r \rightarrow \infty} \frac{\sum_{j=1}^{n+2} N_{n}\left(r, \boldsymbol{a}_{j}, f\right)}{\log r}=\infty .
$$

(a) In particular, if $\boldsymbol{a}_{1}, \ldots, \boldsymbol{a}_{n+1} \in \mathscr{P}$ and if $f$ is of order finite (resp. infinite),

$$
\liminf _{r \rightarrow \infty} \frac{N_{n}\left(r, \boldsymbol{a}_{n+2}, f\right)}{r}>0 \quad\left(\text { resp. } \limsup _{r \rightarrow \infty, r \notin E} \frac{N_{n}\left(r, \boldsymbol{a}_{n+2}, f\right)}{r}>0\right) .
$$

(III) If $f$ is linearly non-degenerate over $C$, then

$$
\bigcup_{j=1}^{n+2}\left\{z \mid\left(\boldsymbol{a}_{j}, f(z)\right)=0\right\} \neq \phi .
$$

(b) In particular, if $\boldsymbol{a}_{1}, \ldots, \boldsymbol{a}_{n+1} \in \mathscr{M}^{1}$,

$$
\lim _{r \rightarrow \infty} N_{n}\left(r, \boldsymbol{a}_{n+2}, f\right) / \log r=\infty .
$$

(IV) If $f$ is linearly non-degenerate over $\mathscr{M}_{o}$ and $0<\rho_{f}<\infty$, then

$$
\limsup _{r \rightarrow \infty} \frac{\log \left(\sum_{j=1}^{n+2} N_{n}\left(r, \boldsymbol{a}_{j}, f\right)\right)}{\log r}=\rho_{f} .
$$

Proof. (I) Suppose that there exist $n+2$ vectors $\boldsymbol{a}_{1}, \ldots, \boldsymbol{a}_{n+2} \in X^{n}(2)$ such that

$$
\limsup _{r \rightarrow \infty} \frac{\sum_{j=1}^{n+2} N_{n}\left(r, \boldsymbol{a}_{j}, f\right)}{T(r, f)}=0 .
$$

Then, for $\boldsymbol{a}_{j}(j=1, \ldots, n+2)$ we obtain that

$$
\limsup _{r \rightarrow \infty} N_{n}\left(r, \boldsymbol{a}_{j}, f\right) / T(r, f)=0 .
$$

This implies that

$$
\delta_{n}\left(\boldsymbol{a}_{j}, f\right)=1 \quad(j=1, \ldots, n+2),
$$

so that $\# \mathscr{D}^{1} \geq n+2$, which contradicts Theorem 2.1(I). (2.7) must hold.

(II) Suppose that there exist $n+2$ vectors $\boldsymbol{a}_{1}, \ldots, \boldsymbol{a}_{n+2} \in X^{n}(2)$ such that

$$
\lim _{r \rightarrow \infty} \frac{\sum_{j=1}^{n+2} N_{n}\left(r, \boldsymbol{a}_{j}, f\right)}{\log r}<\infty .
$$

Then, for any $j=1, \ldots, n+2 \lim _{r \rightarrow \infty} N_{n}\left(r, \boldsymbol{a}_{j}, f\right) / \log r<\infty$. This means that $\boldsymbol{a}_{j} \in \mathscr{P}(j=1, \ldots, n+2)$, so that $\# \mathscr{P} \geq n+2$, which contradicts Theorem 2.1(II). (2.8) must hold.

(III) Suppose that there exist $n+2$ vectors $\boldsymbol{a}_{1}, \ldots, \boldsymbol{a}_{n+2} \in X^{n}(2)$ such that $\left(\boldsymbol{a}_{j}, f\right)$ has no zeros $(j=1, \ldots, n+2)$. Then $\boldsymbol{a}_{j} \in \mathscr{M}^{1}$, so that $\# \mathscr{M}^{1} \geq n+2$, which contradicts Theorem 2.1(III). (2.10) must hold. 
(IV) Suppose that there exist $n+2$ vectors $\boldsymbol{a}_{1}, \ldots, \boldsymbol{a}_{n+2} \in X^{n}(2)$ such that

$$
\limsup _{r \rightarrow \infty} \frac{\log \left(\sum_{j=1}^{n+2} N_{n}\left(r, \boldsymbol{a}_{j}, f\right)\right)}{\log r}<\rho_{f} .
$$

then, for any $j=1, \ldots, n+2$

$$
\limsup _{r \rightarrow \infty} \frac{\log N_{n}\left(r, \boldsymbol{a}_{j}, f\right)}{\log r}<\rho_{f} .
$$

This means that $\boldsymbol{a}_{j} \in \mathscr{B}(j=1, \ldots, n+2)$, so that $\# \mathscr{B} \geq n+2$, which contadicts Theorem 2.1(IV). (IV) must hold.

(II)(a) and (III)(b) We denote by $\mathscr{K}$ any one of $\mathscr{P}$ and $\mathscr{M}^{1}$. We suppose that $f$ is linearly non-degenerate over $\mathscr{M}$, where $\mathscr{M}=\boldsymbol{C}(z)$ when $\mathscr{K}=\mathscr{P}$ and $\mathscr{M}=C$ when $\mathscr{K}=\mathscr{M}^{1}$.

As $\boldsymbol{a}_{1}, \ldots, \boldsymbol{a}_{n+1} \in \mathscr{K}$, from Lemma $2.3, \boldsymbol{a}_{1}, \ldots, \boldsymbol{a}_{n+1}$ are linearly independent, and so $\boldsymbol{a}_{n+2}$ can be represented as a linear combination of $\boldsymbol{a}_{1}, \ldots, \boldsymbol{a}_{n+1}$ with constant coefficients. We suppose without loss of generality that

$$
\boldsymbol{a}_{n+2}=\sum_{j=1}^{k} c_{j} \boldsymbol{a}_{j} \quad\left(c_{j} \neq 0,2 \leq k \leq n+1\right),
$$

from which we obtain the relation

$$
(\boldsymbol{a}, f)=\sum_{j=1}^{k} c_{j}\left(\boldsymbol{a}_{j}, f\right) .
$$

We put $F_{j}=\left(\boldsymbol{a}_{j}, f\right)(j=1, \ldots, k)$ and $F_{o}=(\boldsymbol{a}, f)$. Then, from Lemma 2.2(I) $F_{1}, \ldots, F_{k}$ are linearly independent over $\mathscr{M}$ and from (2.12)

$$
F_{o}=\sum_{j=1}^{k} c_{j} F_{j} \quad\left(c_{j} \neq 0\right) .
$$

Let $g$ be a polynomial such that the functions $F_{1} / g, \ldots, F_{k} / g$ are entire functions without common zeros and put $F=\left[F_{1} / g, \ldots, F_{k} / g\right]$, where $g=1$ when $\mathscr{K}=\mathscr{M}^{1}$.

Then, from Lemma 2.2(I) and (II)(ii), $F$ is transcendental. We note that the functions $F_{1}, \ldots, F_{k}$ have no zeros when $\mathscr{K}=\mathscr{M}^{1}$. From Lemma 2.2(III) applying to $(2.13)$ we have the inequality

$$
\begin{aligned}
T(r, F) & \leq \sum_{j=1}^{k} N_{n}\left(r, \boldsymbol{a}_{j}, f\right)+N_{n}\left(r, \boldsymbol{a}_{n+2}, f\right)+S(r, F) \\
& \leq N_{n}\left(r, \boldsymbol{a}_{n+2}, f\right)+S(r, F),
\end{aligned}
$$

since $\boldsymbol{a}_{j} \in \mathscr{K}(j=1, \ldots, k)$. 
(a) When $\mathscr{K}=\mathscr{P}$. If the order of $f$ is finite, then that of $F$ is also finite from Lemma 2.2(II)(i). From Note 2.1 and (2.14) we have that

$$
\liminf _{r \rightarrow \infty} \frac{N_{n}\left(r, \boldsymbol{a}_{n+2}, f\right)}{T(r, F)} \geq 1
$$

and so from Lemma 2.2(I), (II)(ii) and Lemma 2.4 we have that

$$
\begin{aligned}
\liminf _{r \rightarrow \infty} \frac{N_{n}\left(r, \boldsymbol{a}_{n+2}, f\right)}{r} & \geq \liminf _{r \rightarrow \infty} \frac{N_{n}\left(r, \boldsymbol{a}_{n+2}, f\right)}{T(r, F)} \cdot \liminf _{r \rightarrow \infty} \frac{T(r, F)}{r} \\
& \geq \liminf _{r \rightarrow \infty} \frac{T\left(r, F_{1} / F_{2}\right)}{r}>0 .
\end{aligned}
$$

If the order of $f$ is infinite, from (2.14) we have

$$
\limsup _{r \rightarrow \infty, r \notin E} N_{n}\left(r, \boldsymbol{a}_{n+2}, f\right) / T(r, F) \geq 1,
$$

and so from Lemma 2.2(I), (II)(ii) and Lemma 2.4 we have that

$$
\begin{aligned}
\limsup _{r \rightarrow \infty, r \notin E} \frac{N_{n}\left(r, \boldsymbol{a}_{n+2}, f\right)}{r} & \geq \limsup _{r \rightarrow \infty, r \notin E} \frac{N_{n}\left(r, \boldsymbol{a}_{n+2}, f\right)}{T(r, F)} \cdot \liminf _{r \rightarrow \infty, r \notin E} \frac{T(r, F)}{r} \\
& \geq \liminf _{r \rightarrow \infty, r \notin E} \frac{T\left(r, F_{1} / F_{2}\right)}{r}>0
\end{aligned}
$$

We obtain (2.9).

(b) When $\mathscr{K}=\mathscr{M}^{1}$. Suppose that $N_{n}\left(r, \boldsymbol{a}_{n+2}, f\right)=O(\log r)$. Then, from (2.14) we have that $T(r, F)=S(r, F)$, which is absurd. As $N_{n}\left(r, \boldsymbol{a}_{n+2}, f\right)$ is convex with respect to $\log r$, (2.11) must hold.

Note 2.2. In (III)(b), we can prove the same conclusion as in (2.9).

Note 2.3. A more delicate result is given in [2, Theorem 5.2].

2-3. Example. Let $f, X^{n}(2), \mathscr{D}^{1}, \mathscr{P}, \mathscr{M}^{1}, \mathscr{B}, n$ etc. be as in Introduction and let $\boldsymbol{e}_{j}(j=1, \ldots, n+1)$ be the standard basis of $\boldsymbol{C}^{n+1}$.

Example 2.1. Let $f=\left[1, e^{z}, \ldots, e^{(n-1) z}, e^{n z}\right]$. Then, from [9, (5.3) p. 95],

$$
T(r, f)=(n / \pi) r+O(1),
$$

so that $f$ is transcendental. Let $X^{n}(2)$ be any subset of $\boldsymbol{C}^{n+1} \backslash\{\boldsymbol{0}\}$ containing $\boldsymbol{e}_{j}(j=1, \ldots, n+1)$, which satisfies $(*)$ in Introduction. From Lemma $2.5 f$ is linearly non-degenerate over $\mathscr{M}_{f}$, over $\boldsymbol{C}(z)$, over $\boldsymbol{C}$ and over $\mathscr{M}_{o}$. Further $\left(\boldsymbol{e}_{j}, f\right)$ has no zero for $j=1, \ldots, n+1$ and so

$$
\# \mathscr{D}^{1}=\# \mathscr{P}=\# \mathscr{M}^{1}=\# \mathscr{B}=n+1 .
$$

This shows that Theorem 2.1 is sharp. 
Further, let $\boldsymbol{a}=\left(a_{1}, \ldots, a_{n+1}\right) \in X^{n}(2)$. Then, at least two elements of $a_{1}, \ldots, a_{n+1}$ are different from 0 . Let $a_{j_{1}} \neq 0, \ldots, a_{j_{k}} \neq 0 \quad\left(1 \leq j_{1}<j_{2}<\cdots<\right.$ $\left.j_{k} \leq n+1,2 \leq k \leq n+1\right), a_{j}=0\left(j \neq j_{1}, \ldots, j_{k}\right)$ and put

$$
F=\left[e^{j_{1} z}, \ldots, e^{j_{k} z}\right] .
$$

Then, $T(r, F)=\left(j_{k}-j_{1}\right) r / \pi+O(1)$ so that $F$ is transcendental and of order 1. From Lemma 2.2(III) and Note 2.1 we have the inequality

$$
T(r, F) \leq N_{n}(r, \boldsymbol{a}, f)+O(\log r) .
$$

From this inequality we obtain that

$$
\lim _{r \rightarrow \infty} \frac{\log N_{n}(r, \boldsymbol{a}, f)}{\log r}=1, \quad \liminf _{r \rightarrow \infty} \frac{N_{n}(r, \boldsymbol{a}, f)}{T(r, f)} \geq \frac{1}{n}, \quad \liminf _{r \rightarrow \infty} \frac{N_{n}(r, \boldsymbol{a}, f)}{r} \geq \frac{1}{\pi} .
$$

Example 2.2. Let $g(z)$ be a transcendental entire function such that $T(r, g)=O\left((\log r)^{2}\right)$ and let $f=\left[1, e^{z}, \ldots, e^{(n-1) z}, g(z)\right]$. Then, it is easy to see from Example 2.1 that there are positive constants $a<b<\infty$ satisfying

$$
a r \leq T(r, f) \leq b r,
$$

so that $f$ is transcendetral and of order 1 . Let

$$
X^{n}(2)=\left\{\boldsymbol{e}_{1}, \ldots, \boldsymbol{e}_{n+1}\right\} \cup\left\{\boldsymbol{e}_{1}+\alpha \boldsymbol{e}_{n+1} \mid \alpha \in \boldsymbol{C}, \neq 0\right\} .
$$

Then, $X^{n}(2)$ satisfies $(*)$ in Introduction and

$$
N_{n}\left(r, \boldsymbol{e}_{j}, f\right)=0 \quad(j=1, \ldots, n), \quad N_{n}(r, \boldsymbol{a}, f)=O\left((\log r)^{2}\right),
$$

where $\boldsymbol{a}=\boldsymbol{e}_{1}+\alpha \boldsymbol{e}_{n+1}$.

1) $f$ is linearly degenerate over $\mathscr{M}_{f}, \mathscr{M}_{o}$ and $\# \mathscr{D}^{1}=\# \mathscr{B}=\infty$.

2) $f$ is linearly non-degenerate over $\boldsymbol{C}(z), \# \mathscr{P}=n$ and (2.8) holds, but (2.9) does not hold since this example does not satisfy the condition of Corollary 2.1(II)(a).

Example 2.3. Let $f=\left[1, e^{z}, \ldots, e^{(n-1) z}, z\right](n \geq 2)$. Then, $f$ is transcendental and of order 1 as in Example 2.2. Let $X^{n}(2)$ be the set given in Example 2.2. Then, $X^{n}(2)$ satisfies $(*)$ in Introduction and

$$
N_{n}\left(r, \boldsymbol{e}_{j}, f\right)=0 \quad(j=1, \ldots, n), \quad N_{n}(r, \boldsymbol{a}, f)=O(\log r)
$$

where $\boldsymbol{a}=\boldsymbol{e}_{1}+\alpha \boldsymbol{e}_{n+1}$.

1) $f$ is linearly degenerate over $\boldsymbol{C}(z)$ and $\# \mathscr{P}=\infty$.

2) $f$ is linearly non-degenerate over $\boldsymbol{C}, \# \mathscr{M}^{1}=n$ and (2.10) holds:

Let $\boldsymbol{a}_{1}, \ldots, \boldsymbol{a}_{n+2}$ be any $n+2$ elements in $X^{n}(2)$. Then,

$$
2 \leq \#\left\{\bigcup_{j=1}^{n+2}\left\{z \mid\left(\boldsymbol{a}_{j}, f(z)\right)=0\right\}\right\} \leq n+2 .
$$


The formula (2.11) does not hold as this example does not satisfy the condition of Corollary 2.1(III)(b):

$$
\left.\#\left\{\bigcup_{j=1}^{n+1}\left\{z \mid\left(\boldsymbol{e}_{j}, f(z)\right)=0\right\}\right\}=1 \neq 0 ; \quad \#\{z \mid(\boldsymbol{a}, f(z))=0\}\right\}=1,
$$

where $\boldsymbol{a}=\boldsymbol{e}_{1}+\alpha \boldsymbol{e}_{n+1}$.

\section{Relation between $\mathscr{D}^{1}$ and $\mathscr{B}$}

Let $f, X^{n}(2), n$ etc. be as in Section 1 or 2 .

3-1. Lemma. First of all we shall give the main tool in this section, the fundamental inequality of $\mathrm{H}$. Cartan ([1]) when $N=n$ (Lemma 2.1) and that in general case by E. I. Nochka (see [3]) and then several lemmas to prove theorems.

Lemma 3.1 ([1], see [3]). For any $q$ elements $\boldsymbol{a}_{j}(j=1, \ldots, q)$ of $X$ $(2 N-n+2 \leq q<\infty)$, we have the following inequalities:

(I) $(q-2 N+n-1) T(r, f) \leq \sum_{j=1}^{q} N_{n}\left(r, a_{j}, f\right)+S(r, f)$.

(II) $\sum_{j=1}^{q} \delta_{n}\left(\boldsymbol{a}_{j}, f\right) \leq 2 N-n+1$.

Note 3.1. When $\rho_{f}$ is finite, the error term $S(r, f)$ can be replaced by $O(\log r)$ without exceptional intervals (see the proof of $(\mathrm{I})$ and $[4$, Theorem 2.2(i)]).

DeFinition 3.1. For $0 \leq \alpha<\rho_{f}$, we put

$$
T_{\alpha}(r, f)=\int_{1}^{r} \frac{T(t, f)}{t^{1+\alpha}} d t ; \quad T_{\alpha}(r, \varphi)=\int_{1}^{r} \frac{T(t, \varphi)}{t^{1+\alpha}} d t ;
$$

and

$$
N_{n, \alpha}(r, \boldsymbol{a}, f)=\int_{1}^{r} \frac{N_{n}(t, 0, F)}{t^{1+\alpha}} d t ; \quad \delta_{n, \alpha}(\boldsymbol{a}, f)=1-\limsup _{r \rightarrow \infty} \frac{N_{n, \alpha}(r, \boldsymbol{a}, f)}{T_{\alpha}(r, f)},
$$

where $\varphi$ is a meromorphic function in the plane and $F=(\boldsymbol{a}, f)$ for a vector $\boldsymbol{a} \in \boldsymbol{C}^{n+1} \backslash\{\boldsymbol{0}\}$.

Proposition 3.1 ([8], Proposition 1). For $0 \leq \alpha<\rho_{f}, T_{\alpha}(r, f)$ tends to $+\infty$ when $r \rightarrow \infty$ and we have the followings:

(1) $\lim \sup _{r \rightarrow \infty} \frac{\log T_{\alpha}(r, f)}{\log r}=\rho_{f}-\alpha$; (2) $\lim _{r \rightarrow \infty} \frac{T_{0}(r, f)}{(\log r)^{2}}=+\infty$.

(3) For $0 \leq \alpha<\beta<\rho_{f}$,

$$
\limsup _{r \rightarrow \infty} T_{\alpha}(r, \varphi) / T_{\alpha}(r, f) \geq \limsup _{r \rightarrow \infty} T_{\beta}(r, \varphi) / T_{\beta}(r, f) .
$$

(4) For any $\boldsymbol{a} \in \boldsymbol{C}^{n+1} \backslash\{\boldsymbol{0}\}$ and $0 \leq \alpha<\beta<\rho_{f}, \delta_{n, \alpha}(\boldsymbol{a}, f) \leq \delta_{n, \beta}(\boldsymbol{a}, f)$. 
In particular, if $\boldsymbol{a} \in D^{1}$ or if

$$
\limsup _{r \rightarrow \infty} \frac{\log N_{n}(r, 0, F)}{\log r}=\alpha<\rho_{f},
$$

then for $\beta>\alpha, \delta_{n, \beta}(\boldsymbol{a}, f)=1$.

DEFINITION 3.2.

1) $C(f)=\{\varphi \mid$ meromorphic in $|z|<\infty$ and $T(r, \varphi)=o(T(r, f))(r \rightarrow \infty)\}$;

2) for $0 \leq \alpha<\rho_{f}$

$$
C_{\alpha}(f)=\left\{\varphi \mid \text { meromorphic in }|z|<\infty \text { and } T_{\alpha}(r, \varphi)=o\left(T_{\alpha}(r, f)\right)(r \rightarrow \infty)\right\} .
$$

Proposition 3.2 ([8], Proposition 2). For $0 \leq \alpha<\beta<\rho_{f}$, we have the following:

$$
C(f) \subset C_{\alpha}(f) \subset C_{\beta}(f) .
$$

Proof. (i) Proof of $C(f) \subset C_{\alpha}(f)$. Let $\varphi \in C(f)$. Then, for any positive number $\varepsilon$ there is a positive number $r_{o}$ such that for any $r \geq r_{o}$ we have the inequality

$$
T(r, \varphi) \leq \varepsilon T(r, f),
$$

so that we have the inequality

$$
\frac{T_{\alpha}(r, \varphi)}{T_{\alpha}(r, f)} \leq \frac{T_{\alpha}\left(r_{o}, \varphi\right) /\left\{T_{\alpha}(r, f)-T_{\alpha}\left(r_{o}, f\right)\right\}+\varepsilon}{T_{\alpha}\left(r_{o}, f\right) /\left\{T_{\alpha}(r, f)-T_{\alpha}\left(r_{o}, f\right)\right\}+1},
$$

from which we obtain

$$
\limsup _{r \rightarrow \infty} T_{\alpha}(r, \varphi) / T_{\alpha}(r, f) \leq \varepsilon .
$$

As $\varepsilon$ is arbitrary, we have that

$$
\lim _{r \rightarrow \infty} T_{\alpha}(r, \varphi) / T_{\alpha}(r, f)=0 .
$$

This means that $\varphi$ belongs to $C_{\alpha}(f)$.

(ii) Proof of $C_{\alpha}(f) \subset C_{\beta}(f)$. Let $\varphi \in C_{\alpha}(f)$. Then, for any positive number $\varepsilon$ there is a positive number $r_{o}$ such that for any $r \geq r_{o}$ we have the inequality

$$
T_{\alpha}(r, \varphi) \leq \varepsilon T_{\alpha}(r, f) .
$$

On the other hand the integration by parts gives us the following relations for $\gamma=\beta-\alpha>0$ :

$$
T_{\beta}(r, \varphi)=\gamma \int_{1}^{r} \frac{T_{\alpha}(t, \varphi)}{t^{1+\gamma}} d t+\frac{T_{\alpha}(r, \varphi)}{r^{\gamma}}
$$

and

$$
T_{\beta}(r, f)=\gamma \int_{1}^{r} \frac{T_{\alpha}(t, f)}{t^{1+\gamma}} d t+\frac{T_{\alpha}(r, f)}{r^{\gamma}}
$$


From the above two equations with (3.1) we obtain

$$
\frac{T_{\beta}(r, \varphi)}{T_{\beta}(r, f)} \leq \varepsilon+\gamma \frac{\int_{1}^{r_{o}} \frac{T_{\alpha}(t, \varphi)-\varepsilon T_{\alpha}(t, f)}{t^{1+\gamma}} d t}{\gamma \int_{1}^{r} \frac{T_{\alpha}(t, f)}{t^{1+\gamma}} d t+\frac{T_{\alpha}(r, f)}{r^{\gamma}}} .
$$

As the denominator of the right-hand side tends to $\infty$ for $r \rightarrow \infty$, we obtain that

$$
\limsup _{r \rightarrow \infty} T_{\beta}(r, \varphi) / T_{\beta}(r, f) \leq \varepsilon,
$$

from which we obtain that

$$
\lim _{r \rightarrow \infty} T_{\beta}(r, \varphi) / T_{\beta}(r, f)=0,
$$

since $\varepsilon$ is any positive number. This means that $\varphi \in C_{\beta}(f)$.

Definition 3.3. We put

$$
\mathscr{M}(f)=C(f) \cup\left\{\bigcup_{0 \leq \alpha<\rho_{f}} C_{\alpha}(f)\right\}
$$

LeMma 3.2. The set $\mathscr{M}(f)$ is a field.

Proof. Let $\varphi_{1}, \varphi_{2}$ be in $\mathscr{M}(f)$. Without loss of generality we suppose that $\varphi_{1}, \varphi_{2} \in C_{\beta}$ for some positive number $\beta<\rho_{f}$.

i) We have the relation from the Definition 3.1:

$$
T_{\beta}\left(r, \varphi_{1} \pm \varphi_{2}\right) \leq T_{\beta}\left(r, \varphi_{1}\right)+T_{\beta}\left(r, \varphi_{2}\right)+O(1)=o\left(T_{\beta}(r, f)\right) \quad(r \rightarrow \infty),
$$

which means that $\varphi_{1} \pm \varphi_{2} \in C_{\beta}(f) \subset \mathscr{M}(f)$.

ii) From a property of the characteristic function, we obtain the following inequality

$$
T_{\beta}\left(r, \varphi_{1} \cdot \varphi_{2}^{ \pm 1}\right) \leq T_{\beta}\left(r, \varphi_{1}\right)+T_{\beta}\left(r, \varphi_{2}\right)+O(1)=o\left(T_{\beta}(r, f)\right) \quad(r \rightarrow \infty),
$$

where $\varphi_{2} \neq 0$ when $\pm 1=-1$.

From i) and ii) we have this lemma. plane,

Lemma 3.3. (i)([6], pp. 62-63) For any meromorphic function $h$ in the

$$
\int_{1}^{r} \frac{m\left(t, h^{\prime} / h\right)}{t^{1+\alpha}} d t=O\left(\int_{1}^{r} \frac{\log ^{+} T(t, h)}{t^{1+\alpha}} d t\right) \quad(r \rightarrow \infty, 0<\alpha) .
$$

(ii)(see [6], (21), p. 69) For $0<\alpha<\rho_{f}$

$S_{\alpha}(r, f) \equiv \int_{1}^{r} \frac{S(t, f)}{t^{1+\alpha}} d t=O\left(\int_{1}^{r} \frac{\log ^{+} T(t, f)}{t^{1+\alpha}} d t\right)=o\left(T_{\alpha}(r, f)\right) \quad(r \rightarrow \infty)$. 
Proof (ii). We obtain this relation from the proof of Lemma 2.1(1) for the error term, from the inequality $\left(6^{\prime}\right)$ in [1] and (i) of this lemma.

From now on in this section we suppose that the curve $f=\left[f_{1}, \ldots, f_{n+1}\right]$ is of order $0<\rho_{f} \leq \infty$ and is linearly non-degenerate over $\mathscr{M}(f)$. In this case, Lemma 2.2 holds for $\mathscr{M}=\mathscr{M}(f)$ since we can apply the proof of Lemma 2.2 for $\mathscr{M}=\mathscr{M}(f)$ so that we apply Lemma 2.2 for $\mathscr{M}=\mathscr{M}(f)$ in Lemma 3.4.

Lemma 3.4. Suppose that $\left\{\mathscr{D}^{1} \cup \mathscr{B}\right\} \neq \phi . \quad$ Then, for $1 \leq k \leq \min \left\{\#\left\{\mathscr{D}^{1} \cup \mathscr{B}\right\}\right.$, $n+1\}$, any $k$ vectors in $\left\{\mathscr{D}^{1} \cup \mathscr{B}\right\}$ are linearly independent over $\boldsymbol{C}$.

Proof. (i) When $k=1$. This is trivial since $\mathscr{D}^{1} \cup \mathscr{B} \subset X^{n}(2) \subset \boldsymbol{C}^{n+1} \backslash\{\mathbf{0}\}$. tion.

(ii) When $k=2$. This is also trivial since $\mathscr{D}^{1} \cup \mathscr{B}$ satisfies $(*)$ in Introduc-

(iii) For $2 \leq k \leq \min \left\{\#\left\{\mathscr{D}^{1} \cup \mathscr{B}\right\}-1, n\right\}$, suppose that any $k$ vectors in $\mathscr{D}^{1} \cup \mathscr{B}$ are linearly independent over $\boldsymbol{C}$. We prove that any $k+1$ vectors in $\mathscr{D}^{1} \cup \mathscr{B}$ are linearly independent over $\boldsymbol{C}$.

Suppose to the contrary that there are $k+1$ vectors in $\mathscr{D}^{1} \cup \mathscr{B}$, linearly dependent over $\boldsymbol{C}$. Let $\boldsymbol{a}_{1}, \ldots, \boldsymbol{a}_{k}, \boldsymbol{a}$ be in $\mathscr{D}^{1} \cup \mathscr{B}$ and be linearly dependent over $\boldsymbol{C}$. Then, from the hypothesis of induction we can write

$$
\boldsymbol{a}=\sum_{j=1}^{k} c_{j} \boldsymbol{a}_{j} \quad\left(c_{j} \neq 0,1 \leq j \leq k\right),
$$

from which we obtain the relation

$$
(\boldsymbol{a}, f)=\sum_{j=1}^{k} c_{j}\left(\boldsymbol{a}_{j}, f\right) .
$$

We put

$$
F_{j}=\left(\boldsymbol{a}_{j}, f\right) \quad(j=1, \ldots, k) \quad \text { and } \quad F_{o}=(\boldsymbol{a}, f) .
$$

Then, we obtain that from Lemma $2.2(\mathrm{I}) F_{1}, \ldots, F_{k}$ are linearly independent over $\mathscr{M}(f)$ and that from (3.2)

$$
F_{o}=\sum_{j=1}^{k} c_{j} F_{j} \quad\left(c_{j} \neq 0\right) .
$$

Let $g$ be an entire function such that the functions $F_{1} / g, \ldots, F_{k} / g$ are entire functions without common zeros. We put $F=\left[F_{1} / g, \ldots, F_{k} / g\right]$. Then, $F$ is transcendental from Lemma 2.2(I) and (II)(i),(ii). From (3.3) and Lemma 2.2(III) we obtain the inequality

$$
T(r, F) \leq \sum_{j=1}^{k} N_{n}\left(r, \boldsymbol{a}_{j}, f\right)+N_{n}(r, \boldsymbol{a}, f)+S(r, F) .
$$


(a) When $\mathscr{D}^{1} \cup \mathscr{B}=\mathscr{D}^{1}$. From Lemma 2.2(I), (II)(ii) and (3.4) we obtain the inequality

$$
\begin{gathered}
0<\limsup _{r \rightarrow \infty} \frac{T_{\alpha}\left(r, F_{2} / F_{1}\right)}{T_{\alpha}(r, f)} \leq \sum_{j=1}^{k} \limsup _{r \rightarrow \infty} \frac{N_{n, \alpha}\left(r, \boldsymbol{a}_{j}, f\right)}{T_{\alpha}(r, f)} \\
+\limsup _{r \rightarrow \infty} \frac{N_{n, \alpha}(r, \boldsymbol{a}, f)}{T_{\alpha}(r, f)}+\limsup _{r \rightarrow \infty} \frac{S_{\alpha}(r, f)}{T_{\alpha}(r, f)}=0
\end{gathered}
$$

from Lemma 3.3(ii) and Proposition 3.1(4) since $\boldsymbol{a}_{j}, \boldsymbol{a} \in \mathscr{D}^{1}$. This is a contradiction. This means that any $k+1$ vectors in $\mathscr{D}^{1}$ must be linearly independent over $\boldsymbol{C}$.

(b) When $\mathscr{D}^{1} \cup \mathscr{B}=\mathscr{B}$. Suppose that

$$
\limsup _{r \rightarrow \infty} \frac{\log N_{n}(r, \boldsymbol{a}, f)}{\log r}=\alpha, \quad \text { and } \quad \limsup _{r \rightarrow \infty} \frac{\log N_{n}\left(r, \boldsymbol{a}_{j}, f\right)}{\log r}=\alpha_{j} \quad(j=1, \ldots, k) \text {. }
$$

Then, there is a number $\rho_{f}>\beta>\max \left\{\alpha, \alpha_{j}(j=1, \ldots, k)\right\}$ as $\boldsymbol{a}, \boldsymbol{a}_{j}(j=1, \ldots, k)$ are in $\mathscr{B}$.

From Lemma 2.2(I), (II)(ii) and (3.4) we obtain the inequality

$$
\begin{gathered}
0<\limsup _{r \rightarrow \infty} \frac{T_{\beta}\left(r, F_{2} / F_{1}\right)}{T_{\beta}(r, f)} \leq \sum_{j=1}^{k} \limsup _{r \rightarrow \infty} \frac{N_{n, \beta}\left(r, \boldsymbol{a}_{j}, f\right)}{T_{\beta}(r, f)} \\
+\limsup _{r \rightarrow \infty} \frac{N_{n, \beta}(r, \boldsymbol{a}, f)}{T_{\beta}(r, f)}+\limsup _{r \rightarrow \infty} \frac{S_{\beta}(r, f)}{T_{\beta}(r, f)}=0
\end{gathered}
$$

from Lemma 3.3(ii) and Proposition 3.1(4) since $\boldsymbol{a}_{j}, \boldsymbol{a}$ are of order less than $\beta$. This is a contradiction. This means that any $k+1$ vectors in $\mathscr{B}$ must be linearly independent over $\boldsymbol{C}$.

(c) When $\mathscr{D}^{1} \neq \phi$ and $\mathscr{B} \neq \phi$. We may suppose without loss of generality that $\mathscr{D}^{1}=\left\{\boldsymbol{a}, \boldsymbol{a}_{1}, \ldots, \boldsymbol{a}_{k_{1}}\right\}$ and $\mathscr{B}=\left\{\boldsymbol{a}_{k_{1}+1}, \ldots, \boldsymbol{a}_{k}\right\}$, where $0 \leq k_{1} \leq k-1$.

Suppose that

$$
\limsup _{r \rightarrow \infty} \frac{\log N_{n}\left(r, \boldsymbol{a}_{j}, f\right)}{\log r}=\alpha_{j} \quad\left(j=k_{1}+1, \ldots, k\right) .
$$

Let $\alpha_{j}\left(j=k_{1}+1, \ldots, k\right)<\beta<\rho_{f}$. From Lemma 2.4(I), (II)(ii) and (3.4) we obtain the inequality

$$
\begin{gathered}
0<\limsup _{r \rightarrow \infty} \frac{T_{\beta}\left(r, F_{2} / F_{1}\right)}{T_{\beta}(r, f)} \leq \sum_{j=1}^{k} \limsup _{r \rightarrow \infty} \frac{N_{n, \beta}\left(r, \boldsymbol{a}_{j}, f\right)}{T_{\beta}(r, f)} \\
+\limsup _{r \rightarrow \infty} \frac{N_{n, \beta}(r, \boldsymbol{a}, f)}{T_{\beta}(r, f)}+\limsup _{r \rightarrow \infty} \frac{S_{\beta}(r, f)}{T_{\beta}(r, f)}=0
\end{gathered}
$$

from Lemma 3.3(ii) and Proposition 3.1(4) since $\boldsymbol{a}, \boldsymbol{a}_{j}\left(1 \leq j \leq k_{1}\right)$ are in $\mathscr{D}^{1}$ and $\boldsymbol{a}_{j}\left(k_{1}+1 \leq j \leq k\right)$ are of order less than $\beta$. This is a contradiction. 
This means that any $k+1$ vectors in $\mathscr{D}^{1} \cup \mathscr{B}$ must be linearly independent over $\boldsymbol{C}$.

3-3. Estimates of $\#\left\{D^{1} \cup B\right\}$ and of $\#\left\{\mathscr{D}^{1} \cup \mathscr{B}\right\}$. We shall give two theorems on $D^{1}, \mathscr{D}^{1}, B$ and $\mathscr{B}$

Theorem 3.1. Suppose that $\rho_{f}>0$. Then, $\#\left\{D^{1} \cup B\right\} \leq 2 N-n+1$.

Proof. (a) When $\left\{D^{1} \cup B\right\}=D^{1}$. It is trivial from Lemma 3.1 (II) that $\# D^{1} \leq 2 N-n+1$ in this case.

(b) When $\left\{D^{1} \cup B\right\}=B$. Let $B=\left\{\boldsymbol{a}_{j} \mid j=1, \ldots, q\right\}(\subset X)$,

$$
\limsup _{r \rightarrow \infty} N_{n}\left(r, \boldsymbol{a}_{j}, f\right) / \log r=\alpha_{j} \quad(j=1, \ldots, q)
$$

and $\alpha_{j}<\beta<\rho_{f}(j=1, \ldots, q)$. Supppose that $q>2 N-n+1$. Then from Lemma $3.1(\mathrm{I})$ we obtain the inequality

$$
(q-2 N+n-1) T_{\beta}(r, f) \leq \sum_{j=1}^{q} N_{n, \beta}\left(r, \boldsymbol{a}_{j}, f\right)+S_{\beta}(r, f),
$$

from which we obtain that

$$
o<q-2 N+n-1 \leq \sum_{j=1}^{q} \limsup _{r \rightarrow \infty} \frac{N_{n, \beta}\left(r, \boldsymbol{a}_{j}, f\right)}{T_{\beta}(r, f)}+\limsup _{r \rightarrow \infty} \frac{S_{\beta}(r, f)}{T_{\beta}(r, f)}=0
$$

from Proposition 3.1(4) and Lemma 3.3(ii). This is a contradiction. This means that

$$
\# B \leq 2 N-n+1 .
$$

$q-1)$. Let

(c) When $D^{1}=\left\{\boldsymbol{a}_{j} \mid j=1, \ldots, k\right\}$ and $B=\left\{\boldsymbol{a}_{j} \mid j=k+1, \ldots, q\right\} \quad(1 \leq k \leq$

$$
\limsup _{r \rightarrow \infty} \log N_{n}\left(r, \boldsymbol{a}_{j}, f\right) / \log r=\alpha_{j} \quad(j=k+1, \ldots, q)
$$

and $\alpha_{j}<\beta<\rho_{f}(j=k+1, \ldots, q)$.

Supppose that $q>2 N-n+1$. Then from Lemma 3.1(I) we obtain the inequality

$$
(q-2 N+n-1) T_{\beta}(r, f) \leq \sum_{j=1}^{q} N_{n, \beta}\left(r, \boldsymbol{a}_{j}, f\right)+S_{\beta}(r, f),
$$

from which we obtain that

$$
0<q-2 N+n-1 \leq \sum_{j=1}^{q} \limsup _{r \rightarrow \infty} \frac{N_{n, \beta}\left(r, \boldsymbol{a}_{j}, f\right)}{T_{\beta}(r, f)}+\limsup _{r \rightarrow \infty} \frac{S_{\beta}(r, f)}{T_{\beta}(r, f)}=0
$$


from Proposition 3.1(4) and Lemma 3.3(ii). This is a contradiction. This means that

$$
\#\left\{D^{1} \cup B\right\} \leq 2 N-n+1 .
$$

THEOREM 3.2. Suppose that $\rho_{f}>0$ and $f$ is linearly non-degenerate over $\mathscr{M}(f)$. Then, $\#\left\{\mathscr{D}^{1} \cup \mathscr{B}\right\} \leq n+1$.

Proof. Suppose that $\#\left\{\mathscr{D}^{1} \cup \mathscr{B}\right\} \geq n+2$. Let $\boldsymbol{a}_{1}, \ldots, \boldsymbol{a}_{n+2}$ be in $\mathscr{D}^{1} \cup \mathscr{B}$. Then, from Lemma 3.4 any $n+1$ vectors in $\left\{\boldsymbol{a}_{1}, \ldots, \boldsymbol{a}_{n+2}\right\}$ are linearly independent over $\boldsymbol{C}$. This means that $\boldsymbol{a}_{1}, \ldots, \boldsymbol{a}_{n+2}$ are in general position in $\boldsymbol{C}^{n+1}$. We can apply Lemma 2.1(I) for $N=n$ and $q=n+2$, and fron Proposition 3.1(4) we obtain a contradiction as follows:

$$
1 \leq \sum_{j=1}^{n+2} \limsup _{r \rightarrow \infty} \frac{N_{n, \beta}\left(r, \boldsymbol{a}_{j}, f\right)}{T_{\beta}(r, f)}+\limsup _{r \rightarrow \infty} \frac{S_{\beta}(r, f)}{T_{\beta}(r, f)}=0
$$

for some positive number $\beta<\rho_{f}$. This is a contradiction. We obtain our theorem.

COROLlary 3.1. Under the same condition as in Theorem 3.2, for any vectors $\left\{\boldsymbol{a}_{1}, \ldots, \boldsymbol{a}_{n+2}\right\} \subset X^{n}(2)$,

$$
\limsup _{r \rightarrow \infty} \frac{\sum_{j=1}^{n+2} N_{n}\left(r, \boldsymbol{a}_{j}, f\right)}{T(r, f)}>0
$$

and at least one of the following $n+2$ numbers

$$
\limsup _{r \rightarrow \infty} \log N_{n}\left(r, \boldsymbol{a}_{j}, f\right) / \log r
$$

is equal to $\rho_{f}$.

Proof. Suppose that (3.5) does not hold. Then, for $j=1, \ldots, n+2$

$$
\limsup _{r \rightarrow \infty} N_{n}\left(r, \boldsymbol{a}_{j}, f\right) / T(r, f)=0 .
$$

This means that $\left\{\boldsymbol{a}_{1}, \ldots, \boldsymbol{a}_{n+2}\right\} \subset \mathscr{D}^{1}$. Namely, $\# \mathscr{D}^{1} \geq n+2$, which contradicts Theorem 3.2.

Next, suppose that none of the $n+2$ numbers of (3.6) is not equal to $\rho_{f}$. Then, the $n+2$ numbers

$$
\limsup _{r \rightarrow \infty} \log N_{n}\left(r, \boldsymbol{a}_{j}, f\right) / \log r \quad(j=1, \ldots, n+2)
$$

are smaller than $\rho_{f}$, which means that $\# \mathscr{B} \geq n+2$. This contradicts Theorem 3.2. We obtain our corollary.

3-4. Example. The purpose of this section is to give a holomorphic curve. It is a holomorphic curve $f_{o}$ of positive order and linearly non-degenerate over 
$\mathscr{M}\left(f_{o}\right)$ with a vector $\boldsymbol{a}$ such that $\delta_{n}\left(\boldsymbol{a}, f_{o}\right)=1$ but $\boldsymbol{a}$ is not Borel exceptional for $f_{o}$.

LemMA 3.5. Let $h=g_{1} / g_{2}$ be a non-constant meromorphic function, where $g_{1}$ and $g_{2}$ are entire functons without common zero and we put

$$
f=\left[g_{1}^{n}, g_{1}^{n-1} g_{2}, \ldots, g_{1} g_{2}^{n-1}, g_{2}^{n}\right] .
$$

Then, $T(r, f)=n T(r, h)+O(1)$.

Proof. From the definition of $T(r, f)$ by H. Cartan ([1]),

$$
\begin{aligned}
T(r, f) & =\frac{1}{2 \pi} \int_{0}^{2 \pi} \log \max \left\{\left|g_{1}\right|^{n},\left|g_{1}\right|^{n-1}\left|g_{2}\right|, \ldots,\left|g_{1}\right|\left|g_{2}\right|^{n-1},\left|g_{2}\right|^{n}\right\} d \theta+O(1) \\
& \leq \frac{1}{2 \pi} \int_{0}^{2 \pi} \log \max \left\{\left|g_{1}\right|^{n},\left|g_{2}\right|^{n}\right\} d \theta+O(1) \\
& \leq \frac{n}{2 \pi} \int_{0}^{2 \pi} \log \max \left\{\left|g_{1}\right|,\left|g_{2}\right|\right\} d \theta+O(1)=n T(r, h)+O(1) .
\end{aligned}
$$

On the other hand

$$
\begin{aligned}
T(r, f) & \geq \frac{1}{2 \pi} \int_{0}^{2 \pi} \log \max \left\{\left|g_{1}\right|^{n},\left|g_{2}\right|^{n}\right\} d \theta+O(1) \\
& =\frac{n}{2 \pi} \int_{0}^{2 \pi} \log \max \left\{\left|g_{1}\right|,\left|g_{2}\right|\right\} d \theta+O(1)=n T(r, h)+O(1) .
\end{aligned}
$$

From (3.7) and (3.8) we have our lemma.

Lemma 3.6. Let $h, g_{1}, \ldots, g_{n+1}$ be meromorphic functions in $|z|<\infty$. Then,

$$
T\left(r, \sum_{j=1}^{n+1} g_{j} h^{n+1-j}\right) \leq n T(r, h)+\sum_{j=1}^{n+1} T\left(r, g_{j}\right)+O(1) .
$$

Proof. (a) When $n=1$. From fundamental properties of the characteristic function for meromorphic functions we have the inequality $T\left(r, g_{1} h+g_{2}\right) \leq T\left(r, g_{1} h\right)+T\left(r, g_{2}\right)+O(1) \leq T(r, h)+T\left(r, g_{1}\right)+T\left(r, g_{2}\right)+O(1)$.

(b) We suppose that (3.9) holds when $n=k$, where $k$ is a positive integer. We shall prove that (3.9) holds when $n=k+1$. From the hypothesis of induction we have the inequality:

$$
\begin{aligned}
T\left(r, \sum_{j=1}^{k+2} g_{j} h^{k+2-j}\right) & =T\left(r, \sum_{j=1}^{k+1} g_{j} h^{k+2-j}+g_{k+2}\right) \\
& \leq T\left(r, h \sum_{j=1}^{k+1} g_{j} h^{k+2-j}\right)+T\left(r, g_{k+2}\right)+O(1)
\end{aligned}
$$




$$
\begin{aligned}
& \leq T(r, h)+T\left(r, \sum_{j=1}^{k+1} g_{j} h^{k+2-j}\right)+T\left(r, g_{k+2}\right)+O() \\
& \leq T(r, h)+k T(r, h)+\sum_{j=1}^{k+1} T\left(r, g_{j}\right)+T\left(r, g_{k+2}\right)+O(1) \\
& =(k+1) T(r, h)+\sum_{j=1}^{k+2} T\left(r, g_{j}\right)+O(1) .
\end{aligned}
$$

From (a) and (b) we see that this lemma holds for any positive integer $n$.

LEMMA 3.7. Let $h_{1}(z)=e^{z}, h_{2}(z)=\prod_{n=2}^{\infty}\left(1+z / n(\log n)^{2}\right)$ and we put $h(z)=$ $h_{1}(z) / h_{2}(z)$. Then, $h(z)$ is meromorphic in $|z|<\infty$, of order $1, N(r, h)$ is of order 1 and $\delta(\infty, h)=1$.

\section{Proof. It is known that}

$$
T\left(r, h_{1}\right)=r / \pi
$$

(see [4, p. 7]) and that

$$
n\left(r, 1 / h_{2}\right) \sim r /(\log r)^{2} .
$$

(see [4, p. 29]). We estimate $\log M\left(r, h_{2}\right)$. From (3.11) let $r_{o} \geq 1$ be a positive number such that for any $r \geq r_{o}$

$$
\frac{9}{10} \frac{r}{(\log r)^{2}} \leq n\left(r, \frac{1}{h_{2}}\right) \leq \frac{10}{9} \frac{r}{(\log r)^{2}} .
$$

Since $\sum_{n=2}^{\infty} 1 /\left(n(\log n)^{2}\right)<\infty$, from an inequality in [4, p. 28] and from (3.12) we obtain the inequality for any $r \geq r_{o}$

$$
\begin{aligned}
\log M\left(r, h_{2}\right) & \leq r \int_{0}^{\infty} \frac{n\left(t, 1 / h_{2}\right)}{t(t+r)} d t \\
& =r \int_{r_{o}}^{r} \frac{n\left(t, 1 / h_{2}\right)}{t(t+r)} d t+r \int_{r}^{\infty} \frac{n\left(t, 1 / h_{2}\right)}{t(t+r)} d t+O(1) \\
& \leq \frac{10}{9}\left\{r \int_{r_{o}}^{r} \frac{d t}{(t+r)(\log t)^{2}}+r \int_{r}^{\infty} \frac{d t}{(t+r)(\log t)^{2}}\right\}+O(1) \\
& \leq \frac{10}{9}\left\{\frac{r}{2} \int_{r_{o}}^{r} \frac{d t}{t(\log t)^{2}}+r \int_{r}^{\infty} \frac{d t}{t(\log t)^{2}}\right\}+O(1) \\
& =\frac{10}{9} r\left\{\frac{1}{2} \int_{\log r_{o}}^{\log r} \frac{d u}{u^{2}}+\int_{\log r}^{\infty} \frac{d u}{u^{2}}\right\}+O(1) \\
& =\frac{5 r}{9}\left(\frac{1}{\log r}+\frac{1}{\log r_{o}}\right)+O(1) \quad\left(r \geq r_{o}\right)
\end{aligned}
$$


so that we have the inequality

$$
\begin{aligned}
N\left(r, 1 / h_{2}\right)+O(1) & \leq T\left(r, h_{2}\right) \leq \log M\left(r, h_{2}\right) \\
& \leq \frac{5 r}{9}\left(\frac{1}{\log r}+\frac{1}{\log r_{o}}\right)+O(1) \quad\left(r \geq r_{o}\right)
\end{aligned}
$$

On the other hand, for $r \geq r_{o}$

$$
\begin{aligned}
N\left(r, 1 / h_{2}\right) & =\int_{0}^{r} \frac{n\left(t, 1 / h_{2}\right)}{t} d t=\int_{r_{o}}^{r} \frac{n\left(t, 1 / h_{2}\right)}{t} d t+O(1) \\
& \geq \frac{9}{10} \int_{r_{o}}^{r} \frac{d t}{(\log t)^{2}}+O(1) \geq \frac{9}{10} \frac{\left(r-r_{o}\right)}{(\log r)^{2}}+O(1)
\end{aligned}
$$

From (3.10) and (3.13),

$$
\begin{aligned}
& T(r, h) \leq T\left(r, h_{1}\right)+T\left(r, h_{2}\right)+O(1) \leq \frac{r}{\pi}+\frac{5 r}{9}\left(\frac{1}{\log r}+\frac{1}{\log r_{o}}\right)+O(1) \\
& T(r, h) \geq T\left(r, h_{1}\right)-T\left(r, h_{2}\right)+O(1) \geq \frac{r}{\pi}-\frac{5 r}{9}\left(\frac{1}{\log r}+\frac{1}{\log r_{o}}\right)+O(1)
\end{aligned}
$$

From (3.13), (3.14), (3.15) and (3.16) we obtain that $h$ is of order $1, N(r, h)$ is also of order 1 and $\lim _{r \rightarrow \infty} N(r, h) / T(r, h)=0$, so that $\delta(\infty, h)=1$, but $\infty$ is not Borel exceptional for $h$.

\section{Example 3.1. Let}

$$
f_{o}=\left[h_{1}^{n}, h_{1}^{n-1} h_{2}, \ldots, h_{1} h_{2}^{n-1}, h_{2}^{n}\right]: \boldsymbol{C} \rightarrow P^{n}(\boldsymbol{C}),
$$

where $h_{1}, h_{2}$ are those given in Lemma 3.7. Then $f_{o}$ is a holomorphic curve

(i) of order 1;

(ii) linearly non-degenerate over $\mathscr{M}\left(f_{o}\right)$;

(iii) $\delta_{n}\left(\boldsymbol{e}_{j}, f_{o}\right)=1(j=2, \ldots, n+1)$ and

(iv) $\boldsymbol{e}_{j}(j=2, \ldots, n+1)$ are not Borel exceptional for $f_{o}$.

Proof. (i) From Lemma 3.5, $T\left(r, f_{o}\right)=n T\left(r, h_{1} / h_{2}\right)+O(1)$ and from Lemma $3.7 h_{1} / h_{2}$ is of order 1 , so is $f_{o}$.

(ii) Suppose that there are $g_{1}, \ldots, g_{n+1} \in \mathscr{M}\left(f_{o}\right)$ satisfying

$$
\sum_{j=1}^{n+1} g_{j} h_{1}^{n+1-j} h_{2}^{j-1}=h_{2}^{n}\left\{\sum_{j=1}^{n+1} g_{j} h^{n+1-j}\right\}=0,
$$

where $h=h_{1} / h_{2}$.

(a) When $n=1 . \quad(3.17)$ reduces to

$$
g_{1} h+g_{2}=0 .
$$


Suppose that $g_{1} \neq 0$. Then, from (3.18) $h=-g_{2} / g_{1}$ so that we obtain the inequality

$$
T(r, h) \leq T\left(r, g_{1}\right)+T\left(r, g_{2}\right)+O(1) .
$$

The left-hand side is of order 1 , but the right-hand side is of order less than 1 since $g_{1}, g_{2} \in \mathscr{M}\left(f_{o}\right)$, which is absurd. This means that $g_{1}$ must be equal to 0 , so that $g_{2}=0$ from (3.18).

(b) When $n=k$, we suppose that $g_{1}=\cdots=g_{k+1}=0$ in (3.17). We shall show that when $n=k+1, g_{1}=\cdots=g_{k+2}=0$ in (3.17).

Suppose that $g_{1} \neq 0$ in (3.17) for $n=k+1$. Then, from (3.17) we obtain that

$$
h^{k+1}=-\frac{1}{g_{1}}\left\{\sum_{j=2}^{k+2} g_{j} h^{k+2-j}\right\} .
$$

Applying Lemma 3.6 to this equality we obtain that

$$
T(r, h) \leq \sum_{j=1}^{k+2} T\left(r, g_{j}\right)+O(1),
$$

which is absurd since left-hand side is of order 1 but the right-hand side is of order less than 1 as $g_{1}, \ldots, g_{k+2} \in \mathscr{M}\left(f_{o}\right)$. This means that $g_{1}$ must be equal to 0 . From (3.17) for $n=k+1$, we obtain that

$$
h \sum_{j=1}^{k+1} g_{j+1} h^{k+1-j}=0 .
$$

From the hypothesis of induction, $g_{2}=\cdots=g_{k+2}=0$.

From (a) and (b) we obtain (ii).

(iii) Since

$$
N\left(r, \boldsymbol{e}_{j}, f_{o}\right)=(j-1) N\left(r, 1 / h_{2}\right)=(j-1) N(r, h) \quad(j=2, \ldots, n+1)
$$

and from Lemma 3.5, $T\left(r, f_{o}\right)=n T(r, h)+O(1)$, we obtain from Lemma 3.7 for $j=2, \ldots, n+1$

$$
\delta_{n}\left(\boldsymbol{e}_{j}, f_{o}\right)=1-\limsup _{r \rightarrow \infty} \frac{(j-1) N\left(r, 1 / h_{2}\right)}{T\left(r, f_{o}\right)}=1-\frac{j-1}{n} \limsup _{r \rightarrow \infty} \frac{N(r, h)}{T(r, h)}=1 .
$$

(iv) As in (iii) for $j=2, \ldots, n+1$,

$$
N\left(r, \boldsymbol{e}_{j}, f_{o}\right)=(j-1) N(r, h)
$$

so that from Lemma $3.7, N\left(r, \boldsymbol{e}_{j}, f\right)$ is of order 1 , namely, $\boldsymbol{e}_{j}(j=2, \ldots, n+1)$ are not Borel exceptional for $f_{o}$.

Note 3.1. G. Valiron ([11, p. 73]) writes that it is evident that a Borel exceptional value is not necessary a deficient value since there are meromorphic functions of irregular growth. 


\section{Defect relation}

Let $f, X, X^{n}(k)(k=1, \ldots, n+1), n$ etc. be as in Section 1,2 or 3 such that $2 N-n+1 \leq \min \left\{\# X^{n}(k), \# X\right\}$.

4-1. Defect relation. The fundamental tools are Lemma 2.1 and Lemma 3.1. From Lemma 3.1 we obtain the following

Defect relation. Let $D^{+}=\left\{\boldsymbol{a} \in X \mid \delta_{n}(\boldsymbol{a}, f)>0\right\}$. Then, $D^{+}$is at most countable and we have the inequality

$$
\sum_{\boldsymbol{a} \in D^{+}} \delta_{n}(\{\boldsymbol{a}, f) \leq 2 N-n+1 .
$$

We are interested in a holomorphic curve $f$ for which the defect relation is extremal:

$$
\sum_{\boldsymbol{a} \in D^{+}} \delta_{n}(\boldsymbol{a}, f)=2 N-n+1
$$

As in Introduction, we set $D^{1}=\left\{\boldsymbol{a} \in X \mid \delta_{n}(\boldsymbol{a}, f)=1\right\}$. Then, from (4.1), we have that $\# D^{1} \leq 2 N-n+1$.

For any finite subset $S$ of $X$, we denote by $d(S)$ the dimension of the subspace generated by elements of $S$.

THEOREM 4.A. Suppose that the relation (4.2) holds.

(I) $\left(\left[9\right.\right.$, Theorem 3.2]) If $d\left(D^{1}\right)=n+1$, then $\# D^{1}=2 N-n+1$. then

(II)([12, Theorem 6.1]) If (i) $N>n=2 m(m \in N)$ and (ii) $d\left(D^{1}\right) \leq n$,

$$
\# D^{1}=d\left(D^{1}\right)+N-n .
$$

The last purpose of this paper is to give an example of holomorphic curve for which the defect relation is extremal and to give the defect relation of holomorphic curves which are not extremal in several cases applying these results.

4-2. Preliminaries and lemma. For a non-empty finite subset $S$ of $X$, we denote by $V(S)$ the vector space spanned by elements of $S$ and by $d(S)$ the dimension of $V(S)$. We put

$$
\mathcal{O}=\{S \subset X \mid 0<\# S \leq N+1\} \text {. }
$$

Definition $4.1([12$, Definition 2.1$])$. (I) We put $\lambda=\min _{S \in \mathcal{O}} \frac{d(S)}{\# S}$.
(II) For $R \varsubsetneqq S(R, S \in \mathcal{O})$, we put

$$
\Lambda(R ; S)=\frac{d(S)-d(R)}{\# S-\# R} .
$$

Then, it is known that $0 \leq \Lambda(R ; S) \leq 1$ ([3, p. 67]). 
LeMmA 4.1. Suppose that $\lambda<(n+1) /(2 N-n+1)$.

(I)(see [12, Proposition 2.3(II)(i)]) There exist an integer $p(1 \leq p<$ $(n+1) / 2)$ and a subfamily $\left\{T_{i} \mid 1 \leq i \leq p\right\}$ of $\mathcal{O}$ such that

(i) $\phi=T_{0} \varsubsetneqq T_{1} \varsubsetneqq \cdots \varsubsetneqq T_{p}, d\left(T_{p}\right)<(n+1) / 2$.
(ii) $\Lambda\left(T_{0} ; T_{1}\right)<\Lambda\left(T_{1} ; T_{2}\right)<\cdots<\Lambda\left(T_{p-1} ; T_{p}\right)<\frac{n+1-d\left(T_{p}\right)}{2 N-n+1-\# T_{p}}$.

(II)(see [12, Definition 3.1], [10, Note 2.2], [9, Theorem 3.1]) We put

$$
w(\boldsymbol{a})= \begin{cases}\Lambda\left(T_{i-1} ; T_{i}\right) & \text { if } \boldsymbol{a} \in T_{i} \backslash T_{i-1} \quad(i=1, \ldots, p), \\ \frac{n+1-d\left(T_{p}\right)}{2 N-n+1-\# T_{p}} & \text { if } \quad \boldsymbol{a} \in X \backslash T_{p}\end{cases}
$$

and

$$
h=\frac{2 N-n+1-\# T_{p}}{n+1-d\left(T_{p}\right)} .
$$

Then, the function $w: X \rightarrow(0,1]$ and the constant $h$ have the following properties:

(a) For any $\boldsymbol{a} \in X, 0<h w(\boldsymbol{a}) \leq 1$ and

$$
T_{p}=\{\boldsymbol{a} \in X \mid h w(\boldsymbol{a})<1\} ;
$$

(b-1) For any $Q \subset X$ satisfying (i) $Q \supset T_{p}$ and (ii) $2 N-n+1 \leq \# Q<\infty$,

$$
\# Q-(2 N-n+1)=h\left(\sum_{\boldsymbol{a} \in Q} w(\boldsymbol{a})-n-1\right) ;
$$

(b-2) $\sum_{\boldsymbol{a} \in X}(1-h w(\boldsymbol{a}))=2 N-n+1-h(n+1)$;

(c) $N / n \leq h \leq(2 N-n+1) /(n+1)$;

(d) For any $S \in \mathcal{O}, \sum_{\boldsymbol{a} \in S} w(\boldsymbol{a}) \leq d(S)$.

Suppose that $\lambda<(n+1) /(2 N-n+1)$ and we put

$$
\mathcal{O}_{p}=\left\{S \in \mathcal{O} \mid T_{p} \varsubsetneqq S, d\left(T_{p}\right)<d(S)\right\} .
$$

Then, we have

Proposition 4.1 (see [12, Proposition 2.3(II)(iv)]). For any $S \in \mathcal{O}_{p}$,

$$
\frac{n+1-d\left(T_{p}\right)}{2 N-n+1-\# T_{p}} \leq \frac{d(S)-d\left(T_{p}\right)}{\# S-\# T_{p}} .
$$

Definition 4.2 ([12, Definition 3.1]). We say that

(I) $X$ is of type I if for any $S \in \mathcal{O}_{p}$

$$
h^{-1}=\frac{n+1-d\left(T_{p}\right)}{2 N-n+1-\# T_{p}}<\frac{d(S)-d\left(T_{p}\right)}{\# S-\# T_{p}} .
$$


(II) $X$ is of type II if for some $S \in \mathcal{O}_{p}$

$$
h^{-1}=\frac{n+1-d\left(T_{p}\right)}{2 N-n+1-\# T_{p}}=\frac{d(S)-d\left(T_{p}\right)}{\# S-\# T_{p}} .
$$

We know ([3, p. 68]) that for any $S \in \mathcal{O}, \# S \leq d(S)+N-n$.

Definition 4.3 ([12, p. 357]). For $S \in \mathcal{O}$, we say that $S$ is maximal when

$$
\# S=d(S)+N-n \text {. }
$$

Proposition 4.2 ([3, p. 68]). Let $R, S \in \mathcal{O}$ such that $R \varsubsetneqq S$. If $R$ is maximal, so is $S$.

As a note, we estimate $1-h w(\boldsymbol{a})>0\left(\boldsymbol{a} \in T_{p}\right)$.

Note 4.1. Suppose that $\lambda<(n+1) /(2 N-n+1)$ and that $T_{p}$ is maximal. Then,

$$
1-h w(\boldsymbol{a}) \geq 2 / n(2 N-n) \quad\left(\boldsymbol{a} \in T_{p}\right) .
$$

Proof. From the definition of $w$ (see Lemma 4.1) there is a subset $S$, which may be empty, of $T_{p}$ such that for any $\boldsymbol{a} \in T_{p}$

$$
w(\boldsymbol{a}) \leq \frac{d\left(T_{p}\right)-d(S)}{\# T_{p}-\# S}<h^{-1}=\frac{n+1-d\left(T_{p}\right)}{N+1-d\left(T_{p}\right)},
$$

since $T_{p}$ is maximal, so that we have the inequality

$$
\left(d\left(T_{p}\right)-d(S)\right)\left(N+1-d\left(T_{p}\right)\right)<\left(n+1-d\left(T_{p}\right)\right)\left(\# T_{p}-\# S\right) .
$$

As both terms are integers, we have the inequality

$$
\left(d\left(T_{p}\right)-d(S)\right)\left(N+1-d\left(T_{p}\right)\right) \leq\left(n+1-d\left(T_{p}\right)\right)\left(\# T_{p}-\# S\right)-1,
$$

so that we obtain

$$
\frac{d\left(T_{p}\right)-d(S)}{\# T_{p}-\# S} \cdot \frac{N+1-d\left(T_{p}\right)}{n+1-d\left(T_{p}\right)} \leq 1-\frac{1}{\left(n+1-d\left(T_{p}\right)\right)\left(\# T_{p}-\# S\right)} \leq 1-\frac{2}{n(2 N-n)} .
$$

We obtain this note from this inequality, (4.3) and the inequality

$$
\# T_{p}-\# S \leq \# T_{p}=d\left(T_{p}\right)+N-n<(n+1) / 2+N-n=(2 N-n+1) / 2 .
$$

Lemma 4.2 ([12, Corollary 4.1]). For $f$ and $X$ as in Section 1, we have the inequality
(I) $\sum_{\boldsymbol{a} \in X} w(\boldsymbol{a}) \delta_{n}(\boldsymbol{a}, f) \leq n+1$.
(II) $\sum_{\boldsymbol{a} \in X} \delta_{n}(\boldsymbol{a}, f) \leq(n+1) / \lambda$. 
From the equality ([12, Lemma 5.2]):

$$
\begin{aligned}
2 N-n+1-\sum_{\boldsymbol{a} \in X} \delta_{n}(\boldsymbol{a}, f)= & \sum_{\boldsymbol{a} \in X}(1-h w(\boldsymbol{a}))\left(1-\delta_{n}(\boldsymbol{a}, f)\right) \\
& +h\left(n+1-\sum_{\boldsymbol{a} \in X} w(\boldsymbol{a}) \delta_{n}(\boldsymbol{a}, f)\right)
\end{aligned}
$$

and Lemma 4.2(I) we have the following

Lemma 4.3 ([12, Lemma 6.1]). Suppose that $N>n$. The truncated defect relation for $f$ is extremal:

$$
\sum_{\boldsymbol{a} \in X} \delta_{n}(\boldsymbol{a}, f)=2 N-n+1
$$

if and only if

(i) $(1-h w(\boldsymbol{a}))\left(1-\delta_{n}(\boldsymbol{a}, f)\right)=0 \quad(\boldsymbol{a} \in X)$;

(ii) $\sum_{\boldsymbol{a} \in X} w(\boldsymbol{a}) \delta_{n}(\boldsymbol{a}, f)=n+1$.

Lemma 4.4 ([12, Theorem 5.4]). Suppose that $\lambda<(n+1) /(2 N-n+1)$ and that (i) $X$ is of type $I$ and $T_{p}$ is not maximal or (ii) $X$ is of type II. Then

$$
\sum_{\boldsymbol{a} \in X} \delta_{n}(\boldsymbol{a}, f) \leq 2 N-n+1-\frac{1}{2 n} .
$$

Lemma 4.5 ([12, Corollary 5.1]). Suppose that $N>n=2 m(m \in N) . \quad$ If

$$
\sum_{\boldsymbol{a} \in X} \delta_{n}(\boldsymbol{a}, f)>2 N-n+1-\frac{1}{2 n},
$$

then $\lambda<(n+1) /(2 N-n+1)$.

4-3. Example. In this section we shall give a transcendental holomorphic curve and a set $X^{n}(2)$ in $N$-subgeneral position for which the defect relation is extremal.

Example 4.1. For $n \geq 3$, there are a transcendental holomorphic curve $f$ and a set $X^{n}(2)$ in $N$-subgeneral position satisfying

$$
\sum_{\boldsymbol{a} \in X^{n}(2)} \delta(\boldsymbol{a}, f)=2 N-n+1 .
$$

Proof. Let $d$ be an integer satisfying $2 \leq d \leq(n+1) / 2$ and $p_{1}, \ldots, p_{n+1}$ be $n+1$ polynomials without common zeros and linearly independent over $\boldsymbol{C}$. We put

$$
f=\left[p_{1}, \ldots, p_{d}, p_{d+1} e^{z}, \ldots, p_{n+1} e^{z}\right]
$$


Then, $f$ is linearly non-degenerate over $C$ and $T(r, f) \sim T\left(r, e^{z}\right)$, so that $f$ is transcendental. Let $\boldsymbol{e}_{1}^{\prime}=(1,0, \ldots, 0), \ldots, \boldsymbol{e}_{d}^{\prime}=(0, \ldots, 0,1)$ be the standard basis of $\boldsymbol{C}^{d}$ and let $\boldsymbol{a}_{j}^{\prime}(j=1, \ldots, N-n)$ be vectors in $\boldsymbol{C}^{d}$ such that $\boldsymbol{e}_{1}^{\prime}, \ldots, \boldsymbol{e}_{d}^{\prime}$, $\boldsymbol{a}_{1}^{\prime}, \ldots, \boldsymbol{a}_{N-n}^{\prime}$ are in general position in $\boldsymbol{C}^{d}$. Next, let

$$
\boldsymbol{e}_{d+1}^{\prime}=(1,0, \ldots, 0), \ldots, \boldsymbol{e}_{n+1}^{\prime}=(0, \ldots, 0,1)
$$

be the standard basis of $\boldsymbol{C}^{n+1-d}$ and let $\boldsymbol{b}_{j}^{\prime}(j=1, \ldots, N-n)$ be vectors in $\boldsymbol{C}^{n+1-d}$ such that $\boldsymbol{e}_{d+1}^{\prime}, \ldots, \boldsymbol{e}_{n+1-d}^{\prime}, \boldsymbol{b}_{1}^{\prime}, \ldots, \boldsymbol{b}_{N-n}^{\prime}$ are in general position in $\boldsymbol{C}^{n+1-d}$. We put

$$
\boldsymbol{e}_{j}=\left(\boldsymbol{e}_{j}^{\prime}, \mathbf{0}\right) \quad(1 \leq j \leq d) ; \quad \boldsymbol{e}_{k}=\left(\mathbf{0}, \boldsymbol{e}_{k}^{\prime}\right) \quad(d+1 \leq k \leq n+1)
$$

so that the vectors $\boldsymbol{e}_{1}, \ldots, \boldsymbol{e}_{n+1}$ are the standard basis of $\boldsymbol{C}^{n+1}$, and

$$
\boldsymbol{a}_{j}=\left(\boldsymbol{a}_{j}^{\prime}, \mathbf{0}\right) \in \boldsymbol{C}^{n+1} \quad(1 \leq j \leq N-n) ; \quad \boldsymbol{b}_{k}=\left(\mathbf{0}, \boldsymbol{b}_{k}^{\prime}\right) \in \boldsymbol{C}^{n+1} \quad(1 \leq k \leq N-n) .
$$

Then, the set $X^{n}(2)=\left\{\boldsymbol{e}_{1}, \ldots, \boldsymbol{e}_{n+1}, \boldsymbol{a}_{1}, \ldots, \boldsymbol{a}_{N-n}, \boldsymbol{b}_{1}, \ldots, \boldsymbol{b}_{N-n}\right\}$ is a subset of $\boldsymbol{C}^{n+1} \backslash\{\boldsymbol{0}\}$ in $N$-subgeneral position satisfying $(*)$ in Introduction and

$$
\begin{gathered}
\delta\left(\boldsymbol{e}_{i}, f\right)=1 \quad(i=1, \ldots, n+1) ; \\
\delta\left(\boldsymbol{a}_{j}, f\right)=1 \quad(j=1, \ldots, N-n) ; \quad \delta\left(\boldsymbol{b}_{k}, f\right)=1 \quad(k=1, \ldots, N-n)
\end{gathered}
$$

because

$$
\left(\boldsymbol{e}_{i}, f\right) \quad(i=1, \ldots, d) ; \quad\left(\boldsymbol{a}_{j}, f\right) \quad(j=1, \ldots, N-n)
$$

are polynomials and

$$
\left(\boldsymbol{e}_{i}, f\right) / e^{z} \quad(i=d+1, \ldots, n+1) ; \quad\left(\boldsymbol{b}_{k}, f\right) / e^{z} \quad(k=1, \ldots, N-n)
$$

are polynomials. We have that

$$
\sum_{\boldsymbol{a} \in X^{n}(2)} \delta(\boldsymbol{a}, f)=2 N-n+1 .
$$

4-4. Holomorphic curves with the non-extremal defect relation. Let $f, X, X^{n}(k)(k=1, \ldots, n+1), N>n$ etc. be as in Introduction, Section 1, Section 2 or Section 3.

THEOREM 4.1. Suppose that the sets $X^{n}(k)(k=2, \ldots, n+1)$ are in $N$ subgeneral position. When $n \geq k \geq 2$, we have the defect relation

$$
\sum_{\boldsymbol{a} \in X^{k}(k)} \delta_{k}(\boldsymbol{a}, f) \leq N+N / k
$$

Proof. From the definition of $\lambda$ (Definition 4.1(I)), we have that $k / N \leq \lambda$ due to the definition of $X^{k}(k)$. Then from Lemma 4.2(II) we obtain the inequality

$$
\sum_{\boldsymbol{a} \in X^{k}(k)} \delta_{k}(\boldsymbol{a}, f) \leq N+N / k \leq 2 N-k+1 .
$$


We use $\mathscr{M}_{f}$ as in Section 2.

Lemma 4.6. Suppose that $f$ is linearly non-degenerate over $\mathscr{M}_{f}$. Let $\boldsymbol{a}_{1}, \ldots, \boldsymbol{a}_{k}, \boldsymbol{a}$ be $k+1$ vectors in $D^{1}$ such that $\boldsymbol{a}_{1}, \ldots, \boldsymbol{a}_{k}$ are linearly independent over $\boldsymbol{C}$ and

$$
\boldsymbol{a}=\sum_{j=1}^{k} c_{j} \boldsymbol{a}_{j} \quad\left(c_{j} \neq 0,1 \leq j \leq k\right)
$$

Then, $\boldsymbol{a}=c_{1} \boldsymbol{a}_{1}$.

Proof. Suppose that $k \geq 2$. From (4.4) we obtain the equality

$$
(\boldsymbol{a}, f)=\sum_{j=1}^{k} c_{j}\left(\boldsymbol{a}_{j}, f\right) .
$$

We put

$$
F_{j}=\left(\boldsymbol{a}_{j}, f\right) \quad(j=1, \ldots, k) ; \quad \text { and } \quad F_{o}=(\boldsymbol{a}, f) .
$$

Then, from Lemma $2.1(\mathrm{I})$ for $\mathscr{M}=\mathscr{M}_{f}, F_{1}, \ldots, F_{k}$ are linearly independent over $\mathscr{M}_{f}$ and from (4.5) we obrain the equality

$$
F_{o}=\sum_{j=1}^{k} c_{j} F_{j} \quad\left(c_{j} \neq 0\right) .
$$

Let $g$ be an entire function such that the functions $F_{1} / g, \ldots, F_{k} / g$ are entire functions without common zeros. We put

$$
F=\left[F_{1} / g, \ldots, F_{k} / g\right] .
$$

Then, $F$ is transcendental from Lemma 2.1(I) and Lemma 2.1(II)(ii) for $\mathscr{M}=\mathscr{M}_{f}$. From (4.6) and Lemma $3.1(\mathrm{I})$ for $N=n=k-1$ and $q=k+1$ we obtain the following inequality

$$
\begin{aligned}
T(r, F) & \leq \sum_{j=1}^{k} N_{k-1}\left(r, \boldsymbol{a}_{j}, f / g\right)+N_{k-1}(r, \boldsymbol{a}, f / g)+S(r, F) \\
& \leq \sum_{j=1}^{k} N_{n}\left(r, \boldsymbol{a}_{j}, f\right)+N_{n}(r, \boldsymbol{a}, f)+S(r, f)
\end{aligned}
$$

since

$$
N_{k-1}\left(r, \boldsymbol{a}_{j}, f / g\right) \leq N_{n}\left(r, \boldsymbol{a}_{j}, f\right) \quad(1 \leq j \leq k) ; \quad N_{k-1}(r, \boldsymbol{a}, f / g) \leq N_{n}(r, \boldsymbol{a}, f)
$$

from the definition of these counting functions and

$$
T(r, F) \leq T(r, f)+O(1)
$$


from Lemma $2.1(\mathrm{II})(\mathrm{i})$ for $\mathscr{M}=\mathscr{M}_{f}$. As

$$
T\left(r, F_{1} / F_{2}\right) \leq T(r, F)+O(1)
$$

from Lemma 2.1(II)(ii) for $\mathscr{M}=\mathscr{M}_{f}$, we have from (4.7) and Lemma 2.2(I) for $\mathscr{M}=\mathscr{M}_{f}$ that

$$
\begin{gathered}
0<\limsup _{r \rightarrow \infty, r \notin E} \frac{T\left(r, F_{2} / F_{1}\right)}{T(r, f)} \leq \sum_{j=1}^{k} \limsup _{r \rightarrow \infty} \frac{N_{n}\left(r, \boldsymbol{a}_{j}, f\right)}{T(r, f)} \\
+\limsup _{r \rightarrow \infty} \frac{N_{n}(r, \boldsymbol{a}, f)}{T(r, f)}+\limsup _{r \rightarrow \infty, r \notin E} \frac{S(r, f)}{T(r, f)}=0
\end{gathered}
$$

since $\boldsymbol{a}_{j}, \boldsymbol{a} \in D^{1}$. This is a contradiction. This means that $k$ must be equal to 1 .

THEOREM 4.2. Suppose that $f$ is linearly non-degenerate over $\mathscr{M}_{f}$. Then, $\# D^{1} \leq N+N / n$.

Proof. We have only to prove this inequality when $\# D^{1} \geq N+1$. As $X$ is in $N$-subgeneral position, there are $n+1$ linearly independent vectors $\boldsymbol{a}_{1}, \ldots, \boldsymbol{a}_{n+1}$ in $D^{1}$. There is nothing to prove when $D^{1} \backslash\left\{\boldsymbol{a}_{1}, \ldots, \boldsymbol{a}_{n+1}\right\}=\phi$. We suppose that $D^{1} \backslash\left\{\boldsymbol{a}_{1}, \ldots, \boldsymbol{a}_{n+1}\right\} \neq \phi$. For any $\boldsymbol{a} \in D^{1} \backslash\left\{\boldsymbol{a}_{1}, \ldots, \boldsymbol{a}_{n+1}\right\}$, there are constants $c_{j}(1 \leq j \leq n+1)$ at least one of which is not zero such that

$$
\boldsymbol{a}=\sum_{j=1}^{n+1} c_{j} \boldsymbol{a}_{j} \quad\left(c_{j} \in \boldsymbol{C}\right),
$$

so that from Lemma 4.6 there is only one $c_{j_{1}} \neq 0$ such that $\boldsymbol{a}=c_{j_{1}} \boldsymbol{a}_{j_{1}}$

Let

$$
\#\left(D^{1} \backslash\left\{\boldsymbol{a}_{1}, \ldots, \boldsymbol{a}_{n+1}\right\}\right)=x .
$$

As $X$ is in $N$-subgeneral position, we have the following inequality:

$$
\{(n+1) n+n x\} /(n+1) \leq N,
$$

so that $x \leq N+N / n-(n+1)$ and we have our inequality.

THEOREM 4.3. Suppose that $f$ is linearly non-degenerate over $\mathscr{M}_{f}$, and that $N>n \geq 3$. If $d\left(D^{1}\right)=n+1$, then

$$
\sum_{\boldsymbol{a} \in D^{+}} \delta_{n}(\boldsymbol{a}, f)<2 N-n+1
$$

Proof. Suppose to the contrary that for an $f$

$$
\sum_{\boldsymbol{a} \in D^{+}} \delta_{n}(\boldsymbol{a}, f)=2 N-n+1 .
$$


From Theorem 4.A(I), we have that

$$
D^{+}=D^{1} \text { and } \quad \# D^{1}=2 N-n+1 .
$$

On the other hand from Theorem 4.2

$$
\# D^{1} \leq N+N / n<2 N-n+1
$$

as $n \geq 3$. This is a contradiction. We have our theorem.

THEOREM 4.4. Suppose that $f$ is linearly non-degenerate over $\mathscr{M}_{f}$, that $N>n=2 m(m \in N)$ and that $X^{n}(2)$ is in $N$-subgeneral position. Then,

$$
\sum_{\boldsymbol{a} \in X^{n}(2)} \delta_{n}(\boldsymbol{a}, f)<2 N-n+1 .
$$

Proof. (i) When $d\left(\mathscr{D}^{1}\right)=n+1$. This is a special case of Theorem 4.3.

(ii) When $d\left(\mathscr{D}^{1}\right) \leq n$. Suppose that

$$
\sum_{\boldsymbol{a} \in X^{n}(2)} \delta_{n}(\boldsymbol{a}, f)=2 N-n+1 .
$$

Then, from Theorem 4.A(II), we have that

$$
\# \mathscr{D}^{1}=d\left(\mathscr{D}^{1}\right)+N-n .
$$

From (4.9) we have that $d\left(\mathscr{D}^{1}\right) \geq 2$ since $X^{n}(2)$ is in $N$-subgeneral position satisfying $(*)$ in Introduction. Let $d\left(\mathscr{D}^{1}\right)=k(\geq 2)$ and let $\boldsymbol{b}_{1}, \ldots, \boldsymbol{b}_{k}$ be $k$ linearly independent vectors in $\mathscr{D}^{1}$. Then, from $(4.9) \mathscr{D}^{1} \backslash\left\{\boldsymbol{b}_{1}, \ldots, \boldsymbol{b}_{k}\right\} \neq \phi$. For a vector $\boldsymbol{b} \in \mathscr{D}^{1} \backslash\left\{\boldsymbol{b}_{1}, \ldots, \boldsymbol{b}_{k}\right\}$, there exist constants $c_{j} \neq 0 \in \boldsymbol{C}(j=1, \ldots, k)$ such that $\boldsymbol{b}=\sum_{j=1}^{k} c_{j} \boldsymbol{b}_{j}$.

Then from Lemma 4.6, $k$ must be equal to 1 . This is a contradiction. This implies that (4.8) does not hold. We have our theorem.

4-5. The $\mu_{n}$-defect relation. Let $f, X, X^{n}(k)(k=1, \ldots, n+1), n$, etc. be as in the previous sections. Let $\boldsymbol{a}$ be any vector in $\boldsymbol{C}^{n+1} \backslash\{\boldsymbol{0}\}$. We say that

" $\boldsymbol{a}$ has multiplicity $\mu$ if $(\boldsymbol{a}, f)$ has at least one zero and the zeros of the function $(\boldsymbol{a}, f(z))$ have multiplicity at least $\mu$, while at least one zero has multiplicity $\mu$."

When $(\boldsymbol{a}, f)$ has no zero, we set $\mu=\infty$.

Definition 4.4 ([11, Definition 1.1]). For $\boldsymbol{a} \in \boldsymbol{C}^{n+1} \backslash\{\mathbf{0}\}$ with multiplicity $\mu$ we put

$$
\mu_{n}(\boldsymbol{a}, f)=\left(1-\frac{n}{\mu}\right)^{+}=1-\frac{n}{\max (\mu, n)},
$$

where $a^{+}=\max (a, 0)$ for any neal number $a$. 
We call $\mu_{n}(\boldsymbol{a}, f)$ the $\mu_{n}$-defect of $\boldsymbol{a}$ with respect to $f$. It is known that

$$
0 \leq \mu_{n}(\boldsymbol{a}, f) \leq \delta_{n}(\boldsymbol{a}, f) \leq 1
$$

(see for example [11, Corollary 2.2]).

As a corollary of the Defect relation (4.1), we have the following defect relation for $\mu_{n}(\boldsymbol{a}, f)$ :

Proposition 4.3 (see [5, Corollary (3.B.46)]). For any $\boldsymbol{a}_{1}, \ldots, \boldsymbol{a}_{q} \in X$ $(2 N-n+1 \leq q<\infty)$, we have the following inequality:

$$
\sum_{j=1}^{q} \mu_{n}\left(\boldsymbol{a}_{j}, f\right) \leq 2 N-n+1 .
$$

We call this inequality the $\mu_{n}$-defect relation. Let

$$
M^{+}=\left\{\boldsymbol{a} \in X \mid \mu_{n}(\boldsymbol{a}, f)>0\right\} \quad \text { and } \quad M^{1}=\left\{\boldsymbol{a} \in M^{+} \mid \mu_{n}(\boldsymbol{a}, f)=1\right\} .
$$

Then, $M^{+} \subset D^{+}$by (4.10) and we have the defect relation:

$$
\sum_{\boldsymbol{a} \in X} \mu_{n}(\boldsymbol{a}, f) \leq 2 N-n+1
$$

from Proposition 4.3. If the equality holds in (4.11):

$$
\sum_{\boldsymbol{a} \in X} \mu_{n}(\boldsymbol{a}, f)=2 N-n+1,
$$

then, by (4.1), (4.10) and (4.11) we have

$$
\mu_{n}(\boldsymbol{a}, f)=\delta_{n}(\boldsymbol{a}, f) \quad\left(\boldsymbol{a} \in M^{+}\right)
$$

and so $M^{+}=D^{+}$.

Further we put (see Introduction)

$$
\mathscr{M}^{+}=\left\{\boldsymbol{a} \in X^{n}(2) \mid \mu_{n}(\boldsymbol{a}, f)>0\right\} \quad \text { and } \quad \mathscr{M}^{1}=\left\{\boldsymbol{a} \in \mathscr{M}^{+} \mid \mu_{n}(\boldsymbol{a}, f)=1\right\} .
$$

The main purpose of this section is to estimate

$$
\sum_{\boldsymbol{a} \in X^{n}(2)} \mu_{n}(\boldsymbol{a}, f)
$$

Proposition 4.4. (I)([9, Theorem 4.2 and Proposition 4.2]) (i) If $d\left(M^{1}\right)=n+1$, then, $M^{+}=M^{1}$. (ii) $\# M^{1} \leq N+N / n$.

$($ II $)([11$, Note 3.1$]) \# M^{+}+n \# M^{1} \leq(n+1)(2 N-n+1)$. In particular, $\# M^{+}$is finite. 
Lemma 4.7 ([10, in the proof of Theorem 3.2]). Suppose that $N>n \geq 1$ and that (4.12) holds. If $d\left(M^{1}\right) \geq 1$, then

$$
d\left(M^{1}\right)=\sum_{\boldsymbol{a} \in M^{1}} w(\boldsymbol{a})
$$

Lemma 4.8. Suppose that (i) $N>n \geq 2$ and that (ii) (4.12) holds. If $d\left(M^{1}\right) \geq 1$, then

$$
h=\left(2 N-n+1-\# M^{1}\right) /\left(n+1-d\left(M^{1}\right)\right) .
$$

Proof. From Lemma 4.3 and (4.13) we have that

$$
h w(\boldsymbol{a})=1 \quad\left(\boldsymbol{a} \in M^{+}-M^{1}\right)
$$

and

$$
\sum_{\boldsymbol{a} \in M^{+}} w(\boldsymbol{a}) \mu_{n}(\boldsymbol{a}, f)=n+1 .
$$

From (4.15) and Lemma 4.7,

$$
\begin{aligned}
n+1 & =\sum_{\boldsymbol{a} \in M^{+}} w(\boldsymbol{a}) \mu_{n}(\boldsymbol{a}, f)=\sum_{\boldsymbol{a} \in M^{1}} w(\boldsymbol{a})+\sum_{\boldsymbol{a} \in M^{+}-M^{1}} w(\boldsymbol{a}) \mu_{n}(\boldsymbol{a}, f) \\
& =d\left(M^{1}\right)+\sum_{\boldsymbol{a} \in M^{+}-M^{1}} w(\boldsymbol{a}) \mu_{n}(\boldsymbol{a}, f) .
\end{aligned}
$$

From (4.14)

$$
\begin{aligned}
h\left(n+1-d\left(M^{1}\right)\right) & =\sum_{\boldsymbol{a} \in M^{+}-M^{1}} h w(\boldsymbol{a}) \mu_{n}(\boldsymbol{a}, f) \\
& =\sum_{\boldsymbol{a} \in M^{+}-M^{1}} \mu_{n}(\boldsymbol{a}, f)=2 N-n+1-\# M^{1} .
\end{aligned}
$$

We have our lemma since $d\left(M^{1}\right) \leq n$ from Proposition 4.4(I),(II) and (4.12).

Lemma 4.9. Suppose that (i) $N>n=2 m(m \in N)$ and that (ii) (4.12) holds. Then, $\lambda<(n+1) /(2 N-n+1)$.

Proof. From (4.13) and Lemma 4.5 we have $\lambda<(n+1) /(2 N-n+1)$.

Lemma 4.10. Suppose that (i) $N>n \geq 2$ and that (ii) (4.12) holds. If $\lambda<(n+1) /(2 N-n+1)$, then $X$ is of type I, $T_{p}$ is maximal and $M^{1}=T_{p}$ so that

$$
\# M^{1}=d\left(M^{1}\right)+N-n, \quad d\left(M^{1}\right)<(n+1) / 2 .
$$


Proof. First of all we note that $M^{1} \in \mathcal{O}$ since $d\left(M^{1}\right) \leq n$ from Proposition 4.4(I), (4.12), (4.13) and Theorem 4.A(I). By Lemma 4.3 and (4.13) we have

$$
T_{p} \subset M^{1} .
$$

From (4.12), (4.13) and Lemma 4.4, we have that $X$ is of type $\mathrm{I}, T_{p}$ is maximal and so $M^{1}$ is also maximal from (4.16) and Proposition 4.2:

$$
\# M^{1}=d\left(M^{1}\right)+N-n
$$

Further, as $d\left(M^{1}\right) \geq 1$ from (4.17), by Lemma 4.8 and Lemma 4.1(II) we have

$$
h=\frac{2 N-n+1-\# T_{p}}{n+1-d\left(T_{p}\right)}=\frac{2 N-n+1-\# M^{1}}{n+1-d\left(M^{1}\right)} .
$$

If $T_{p} \varsubsetneqq M^{1}$, then $d\left(T_{p}\right)<d\left(M^{1}\right)$ and

$$
\frac{2 N-n+1-\# T_{p}}{n+1-d\left(T_{p}\right)}=\frac{\# M^{1}-\# T_{p}}{d\left(M^{1}\right)-d\left(T_{p}\right)} .
$$

As $M^{1} \in \mathcal{O}$, this means that $X$ is of type II from Definition 4.2, which is a contradiction. We have that $T_{p}=M^{1}$. From Lemma 4.1(I) we obtain that $d\left(M^{1}\right)=d\left(T_{p}\right)<(n+1) / 2$.

Theorem 4.5. Suppose that $d\left(\mathscr{M}^{1}\right) \geq 1, N>n \geq 2$ and that $X^{n}(2)$ is in $N$-subgeneral position. Then,

$$
\sum_{\boldsymbol{a} \in X^{n}(2)} \mu_{n}(\boldsymbol{a}, f)<2 N-n+1
$$

Proof. Suppose to the contrary that

$$
\sum_{\boldsymbol{a} \in X^{n}(2)} \mu_{n}(\boldsymbol{a}, f)=2 N-n+1
$$

Then, from Lemma 4.8, we have that

$$
h=\left(2 N-n+1-\# \mathscr{M}^{1}\right) /\left(n+1-d\left(\mathscr{M}^{1}\right)\right) .
$$

From Lemma 4.1(II)(c),

$$
\left(2 N-n+1-\# \mathscr{M}^{1}\right) /\left(n+1-d\left(\mathscr{M}^{1}\right)\right) \leq(2 N-n+1) /(n+1),
$$

which reduces to the inequality

$$
d\left(\mathscr{M}^{1}\right)<\frac{2 N-n+1}{n+1} d\left(\mathscr{M}^{1}\right) \leq \# \mathscr{M}^{1}
$$

and we have that $\# \mathscr{M}^{1} \geq d\left(\mathscr{M}^{1}\right)+1$.

1) When $d\left(\mathscr{M}^{1}\right)=1$. It is trivial that there are two vectors $\boldsymbol{a}$ and $\boldsymbol{b}$ in $\mathscr{M}^{1}$ satisfying $\boldsymbol{a}=c \boldsymbol{b}\left(c \neq 0\right.$, constant). This is absurd since $\boldsymbol{a}, \boldsymbol{b} \in X^{n}(2)$. 
2) When $d\left(\mathscr{M}^{1}\right)=d \geq 2$. We suppose that $\boldsymbol{b}_{1}, \ldots, \boldsymbol{b}_{d}$ are linearly independent vectors belonging to $\mathscr{M}^{1}$. Then, any vector $\boldsymbol{a} \in \mathscr{M}^{1} \backslash\left\{\boldsymbol{b}_{1}, \ldots, \boldsymbol{b}_{d}\right\}$ can be represented by $\boldsymbol{b}_{1}, \ldots, \boldsymbol{b}_{d}$ as a linear combination over $\boldsymbol{C}$ :

$$
\boldsymbol{a}=c_{1} \boldsymbol{b}_{1}+\cdots+c_{d} \boldsymbol{b}_{d} .
$$

From this relation

$$
(\boldsymbol{a}, f)=\sum_{v=1}^{d} c_{v}\left(\boldsymbol{b}_{v}, f\right) .
$$

As $\boldsymbol{a}, \boldsymbol{b}_{1}, \ldots, \boldsymbol{b}_{d}$ are in $\mathscr{M}^{1}$, from (4.18) we obtain that there is an integer $k(1 \leq k \leq d)$ such that

$$
(\boldsymbol{a}, f)=c_{k}\left(\boldsymbol{b}_{k}, f\right) \quad\left(c_{k} \neq 0\right)
$$

due to a Borel's theorem (see $\left[1,1^{o}\right.$, p. 19]). This relation reduces to $\boldsymbol{a}=c_{k} \boldsymbol{b}_{k}$ since $f$ is linearly non-degenerate over $\boldsymbol{C}$. This is absurd since $\boldsymbol{a}, \boldsymbol{b}_{k} \in X^{n}(2)$.

From 1) and 2) our theorem must hold.

Theorem 4.6. Suppose that $N>2 m(m \in N)$ and $X^{n}(2)$ is in $N$-subgeneral position. Then

$$
\sum_{\boldsymbol{a} \in X^{n}(2)} \mu_{n}(\boldsymbol{a}, f)<2 N-n+1
$$

Proof. We suppose to the contrary that there exist $f, X^{n}(2)$ such that

$$
\sum_{\boldsymbol{a} \in X^{n}(2)} \mu_{n}(\boldsymbol{a}, f)=2 N-n+1
$$

Then, from Lemma $4.9, \lambda<(n+1) /(2 N-n+1)$, so that from Lemma 4.10 $\mathscr{M}^{1}$ satisfies

$$
\# \mathscr{M}^{1}=d\left(\mathscr{M}^{1}\right)+N-n ; \quad d\left(\mathscr{M}^{1}\right)<(n+1) / 2 .
$$

This means that $d\left(\mathscr{M}^{1}\right) \geq 1$. From Theorem 4.5, we have that (4.19) does not hold. We have our theorem.

Acknowledgement. The author was supported in part by Grant-in-Aid for General Scientific Reserch (No. (C) 22540233 (Prof. K. Ishizaki)), Japan Society for the Promotion of Science and Culture during the preparation of this paper.

\section{REFERENCES}

[ 1] H. CARTAN, Sur les combinaisons linéaires de $p$ fonctions holomorphes données, Mathematica(Cluj) 7 (1933), 5-31.

[2] P. Corvaja AND J. Noguchi, A new unicity theorem and Erdös problem for polarized semiabelian varieties, to appear in Math. Ann. 
[ 3 ] H. Fusimoto, Value distribution theory of the Gauss map of minimal surfaces in $\boldsymbol{R}^{m}$, Aspects of math. E21, Vieweg 1993.

[4] W. K. Hayman, Meromorphic functions, Oxford at the Clarendon Press, 1964.

[5] S. Kobayashi, Hyperbolic complex spaces, Springer-Verlag, Berlin-Heidelberg-NewYork, 1998.

[6] R. Nevanlinna, Le théorème de Picard-Borel et la théorie des fonctions méromorphes, Gauthier-Villars, Paris, 1929.

[7] E. I. Nochka, On the theory of meromorphic curves, Dokl. Akad. Nauk SSSR 269 (1983), 547-552 (in Russian).

[8] N. TodA, On holomorphic curves extremal for the truncated defect relation and some applications, Proc. Japan Acad., Ser.A. 81 (2005), 99-104.

[9] N. Toda, On holomorphic curves extremal for the truncated defect relation, Proc. Japan Acad., Ser.A. 82 (2006), 18-23.

[10] N. Toda, On holomorphic curves extremal for the $\mu_{n}$-defect relation, Kodai Math. J. 30 (2007), 111-130.

[11] N. TodA, A generalization of Nochka weight function, Proc. Japan Acad., Ser.A. 83 (2007), 170-175.

[12] N. Toda, On the truncated defect relation for holomorphic curves, Kodai Math. J. 32 (2009), 352-389.

[13] G. Valiron, Remarques sur les valeurs exceptionnelles des fonctions meromorphes, Rend. Circ. Mat. Palermo. 57 (1933), 71-86.

[14] H. WeYL, Meromorphic functions and analytic curves, Ann. math. studies 12, Princeton Univ. Press, Princeton, N.J., 1943.

\author{
Nobushige Toda \\ 401-C1113 \\ Hoshigaokayamate, Chikusa-Ku \\ NAGOYA 464-0808 \\ JAPAN \\ E-mail: toda1113@s6.wh.qit.ne.jp
}

